\title{
Thermal Cope reaction of 2-azetidinone-tethered 1,5-dienes: synthesis of tetrahydroazocinones
}

\author{
Pedro Almendros, ${ }^{\text {a* }}$ Cristina Aragoncillo, ${ }^{\mathrm{b}}$ Gema Cabrero, ${ }^{\mathrm{b}}$ Ricardo Callejo, ${ }^{\mathrm{b}}$ Rocío \\ Carrascosa, ${ }^{b}$ Amparo Luna, ${ }^{b}$ Teresa Martínez del Campo, ${ }^{b}$ M. Carmen Pardo, ${ }^{b}$ M. Teresa \\ Quirós, ${ }^{b}$ M. Carmen Redondo, ${ }^{\text {b Carolina Rodríguez-Ranera, }{ }^{b} \text { Alberto Rodríguez-Vicente, }}$ \\ and M. Pilar Ruiz \\ ${ }^{a}$ Instituto de Química Orgánica General, CSIC, Juan de la Cierva 3, 28006-Madrid, Spain \\ ${ }^{b}$ Departamento de Química Orgánica I, Facultad de Química, Universidad Complutense de \\ Madrid, 28040-Madrid, Spain \\ E-mail:Palmendros@iqog.csic.es
}

Dedicated to Prof. Benito Alcaide on the occasion of his $60^{\text {th }}$ birthday

\begin{abstract}
We report herein full details of the first example of a Cope rearrangement in which the C3-C4 bond of the $\beta$-lactam nucleus is the central bond of the 1,5-hexadiene system, thus providing an easy and efficient entry to novel, and in some cases optically pure functionalized azocinones.
\end{abstract}

Keywords: Azocinones, Cope reaction, $\beta$-lactams, rearrangements

\section{Introduction}

Eight-membered nitrogen heterocycles are constituents of a number of compounds with interesting pharmacological properties. ${ }^{1}$ In particular, eight-membered-ring lactams (2azocanones) are significant from a medicinal chemistry perspective. However, in contrast to their congeners with a smaller ring size, eight-membered-ring lactams are relatively rare structural motifs in natural products. ${ }^{2}$ Tailored biologically active compounds of this type are mostly benzo-annulated or fused with other heterocycles. ${ }^{3}$ With at least one endocyclic $\mathrm{C}=\mathrm{C}$ double bond (i.e. hexahydroazocinones), they adopt a conformation that makes them attractive peptide building blocks, since they are known to mimic a dipeptide $\beta$-turn. ${ }^{4}$ Unfortunately, cyclization to medium-sized heterocycles is often slow and is hampered by the unfavorable enthalpies (strain in medium rings) and entropies (probability of the chain ends meeting) of the reaction. ${ }^{5}$

On the other hand, the thermal $\pi^{2} s+\sigma^{2} s+\pi^{2} s$ (Cope) rearrangement of cis-1,2divinylcyclobutane has been developed into a useful and widely applied tool for the synthesis of 
cyclooctane derivatives. ${ }^{6}$ Driven by the release of cyclobutane ring strain, this Cope rearrangement often proceeds at significantly lower temperatures $\left(60-140^{\circ} \mathrm{C}\right)$ than analogous reactions involving acyclic 1,5 -dienes.

The 2-azetidinone ( $\beta$-lactam) skeleton is well established as the key pharmacophore of $\beta$ lactam antibiotics, the most widely employed class of antibacterial agents. ${ }^{7}$ In addition, there are many important nonantibiotic uses of 2-azetidinones in fields ranging from enzyme inhibition ${ }^{8}$ to gene activation. ${ }^{9}$ Apart from their clinical interest, the use of $\beta$-lactams as versatile synthons for the preparation of compounds of biological relevance, such as $\alpha$ - and $\beta$-amino acids, alkaloids, heterocycles, and taxoids, is now well established. ${ }^{10}$ In spite of its synthetic potential, the behavior of the 2-azetidinone nucleus under thermal conditions remains still almost unknown. ${ }^{11}$ Following our commitment in $\beta$-lactam and heterocyclic chemistry, ${ }^{12}$ in this paper we report full details of the thermally induced [3,3] sigmatropic (Cope) rearrangement of $\beta$-lactams having alkenyl groups both at the C3 and C4 positions that fully confirms our earlier conclusions, ${ }^{13}$ and establishes a versatile route to a variety of racemic and enantiopure eight-membered lactams. A brief retrosynthetic analysis for the tetrahydroazocinone unit is illustrated in Scheme 1.

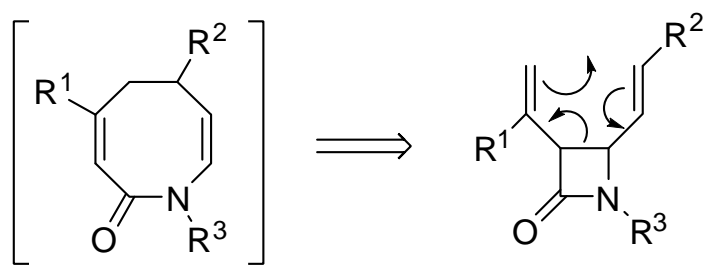

\section{Scheme 1}

\section{Results and Discussion}

Starting 4-formyl $\beta$-lactams 1 were prepared following literature methods. ${ }^{14}$ Rearrangement precursors, 2-azetidinone-tethered dienes $\mathbf{2 - 4}$, were prepared both in the racemic and in optically pure forms using standard methodology. Racemic compounds $\mathbf{2 a - h}$ were obtained as single $E$ isomers or as an $E / Z$ mixture by Wittig olefination of racemic cis-4-formyl $\beta$-lactams $\mathbf{1 a}$ and $\mathbf{1 b}$ (Table 1). Racemic compounds $\mathbf{3 a}$ and $\mathbf{3 b}$ and enantiomerically pure divinyl- $\beta$-lactams $\mathbf{4}$ were prepared as single cis-diastereoisomers from the appropriate cinnamylideneimine, derived from benzylamine or $R-(+)$ - $\alpha$-methylbenzylamine, respectively, through Staudinger reaction with the corresponding system acid chloride/triethylamine, in refluxing dichloromethane (Schemes 2 and 3). ${ }^{15}$ Compounds $\mathbf{4 a}$ and $\mathbf{4 b}$ were obtained as a mixture of diastereoisomers with opposite configuration at the $\mathrm{C} 3$ and $\mathrm{C} 4$ stereocenters of the $\beta$-lactam ring. These isomers were easily separated by flash chromatography. On the basis of the reported data for related $\beta$-lactam systems concerning the ${ }^{1} \mathrm{H}$ NMR chemical shift of the signal corresponding to the exocyclic methine proton $\mathrm{H} 1$ ' attached to $\mathrm{N} 1$, configuration $(1 ' R, 3 S, 4 R)$ and $(1 ' R, 3 R, 4 S)$ was assigned for 
major $(\alpha)$ and minor $(\beta)$ isomers, respectively. ${ }^{16}$ The preferred conformations together with the chemical shifts for protons H1' are depicted in Figure 1.

Table 1. Synthesis of 2-azetidinone-tethered dienes $\mathbf{2}^{a}$

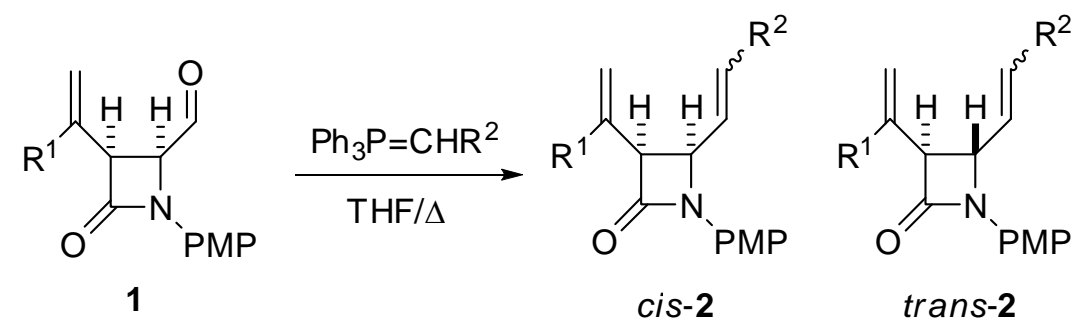

\begin{tabular}{ccccccc}
\hline Aldehyde & $\mathrm{R}^{1}$ & $\mathrm{R}^{2}$ & Diene & cis/trans & Z/E & Yield (\%) \\
\hline$( \pm)-\mathbf{1 a}$ & $\mathrm{H}$ & $\mathrm{CO}_{2} \mathrm{Me}$ & $( \pm)-2 \mathbf{a}$ & $100 /-$ & $-/ 100$ & 82 \\
$( \pm)-\mathbf{1 b}$ & $\mathrm{Me}$ & $\mathrm{CO}_{2} \mathrm{Me}$ & $( \pm)-\mathbf{2 b}$ & $100 /-$ & $-/ 100$ & 92 \\
$( \pm)-\mathbf{1 a}$ & $\mathrm{H}$ & $\mathrm{COMe}$ & $( \pm)-2 \mathbf{c}$ & $100 /-$ & $-/ 100$ & 68 \\
$( \pm)-\mathbf{1 b}$ & $\mathrm{Me}$ & $\mathrm{COMe}$ & $( \pm)-\mathbf{2 d}$ & $100 /-$ & $-/ 100$ & 65 \\
$( \pm)-\mathbf{1 a}$ & $\mathrm{H}$ & $\mathrm{Ph}$ & $( \pm)-2 \mathbf{e}$ & $35 / 65$ & $-/ 100$ & $30 / 46^{b}$ \\
$( \pm)-\mathbf{1 b}$ & $\mathrm{Me}$ & $\mathrm{Ph}$ & $( \pm)-\mathbf{2 f}$ & $85 / 15$ & $40 / 60$ & $91^{c}$ \\
$( \pm)-\mathbf{1 a}$ & $\mathrm{H}$ & $\mathrm{CN}$ & $\mathbf{( \pm ) - 2 g}$ & $100 /-$ & $40 / 60$ & $35 / 55^{d}$ \\
$( \pm)-\mathbf{1 b}$ & $\mathrm{Me}$ & $\mathrm{CN}$ & $\mathbf{( \pm ) - 2 h}$ & $100 /-$ & $56 / 44$ & $55 / 34^{d}$ \\
\hline
\end{tabular}

${ }^{a} \mathrm{PMP}=4-\mathrm{MeOC}_{6} \mathrm{H}_{4}$. The assignment of the cis/trans stereochemistry to $\beta$-lactams was based on the observed coupling constants for methine protons H3 and H4 (cis ca. $2.0 \mathrm{~Hz}$; trans ca. 5.0 Hz). ${ }^{b}$ Yields for isomers cis and trans, respectively. The reaction was carried out at room temperature. ${ }^{c}$ Yields for the inseparable mixture of isomers E-cis, Z-cis, and E-trans (45:40:15). The reaction was carried out at room temperature. ${ }^{d}$ Yields for chromatographically separable isomers $Z$ and $E$, respectively.
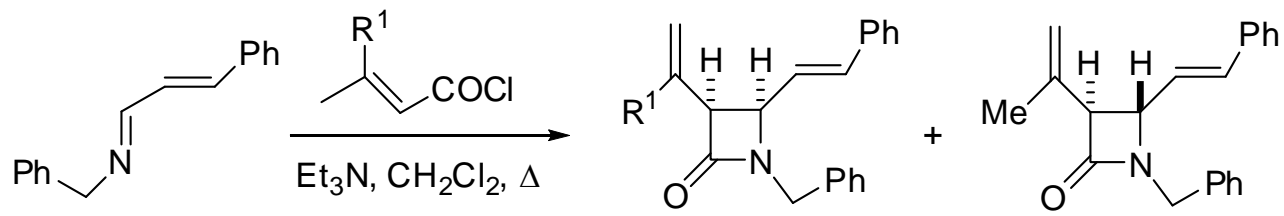

$$
\begin{aligned}
& \mathrm{R}^{1}=\mathrm{H} \text { : cis-( } \pm \text { )-3a (56\%) } \\
& \mathrm{R}^{1}=\mathrm{Me} \text { : cis- }( \pm)-\mathbf{3 b} \quad(54 \%) \quad \text { trans- }( \pm)-\mathbf{3 b} \quad(3 \%)
\end{aligned}
$$

\section{Scheme 2}




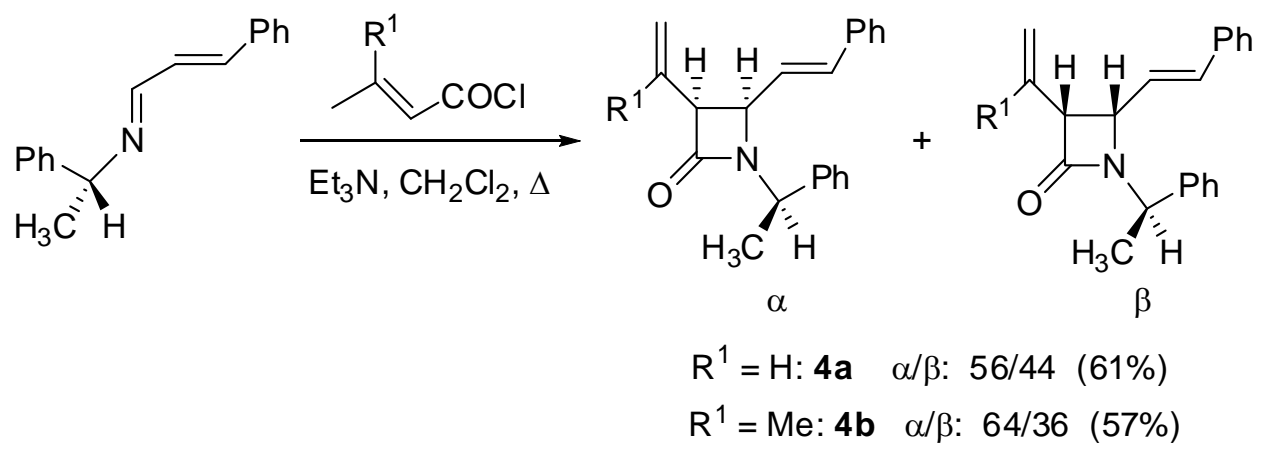

\section{Scheme 3}

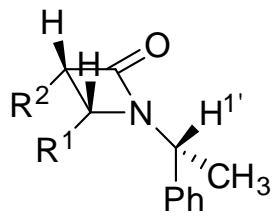

$1^{\prime} R, 3 S, 4 R$

(major isomer $\alpha$ )

(+)-4a- $\alpha: \delta \mathrm{H}^{\prime}=4.92 \mathrm{ppm}$

$(-)-4 b-\alpha: \delta H 1^{\prime}=5.05$ ppm

$\mathrm{R}^{1}:(E) \mathrm{CH}=\mathrm{CHPh} ; \mathbf{4 a}: \mathrm{R}^{2}: \mathrm{CH}=\mathrm{CH}_{2} ; \mathbf{4 b}: \mathrm{R}^{2}: \mathrm{C}(\mathrm{Me})=\mathrm{CH}_{2}$

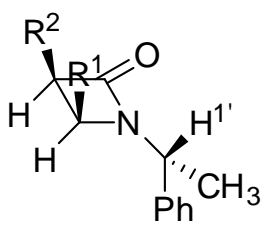

$1^{\prime} R, 3 R, 4 S$

(minor isomer $\beta$ )

$(+)-4 a-\beta: \delta H 1^{\prime}=4.61 \mathrm{ppm}$

$(+)-4 b-\beta: \delta \mathrm{H}^{\prime}=4.60 \mathrm{ppm}$

Figure 1

Having obtained the starting substrates, the next stage was set to carry out the tetrahydroazocinone formation. In an initial reaction, a solution of diene $\mathbf{2 a}$ in toluene was heated at $120^{\circ} \mathrm{C}$ in a sealed tube for 2 hours. Analysis by ${ }^{1} \mathrm{H}$ NMR spectroscopy revealed a quantitative conversion $(>97 \%)$ to the desired tetrahydroazocinone 5a. Next, we tried the same reaction in refluxing toluene, with similar results after 4 hours. According to the strategy outlined above, 2azetidinone-tethered dienes $\mathbf{2} \mathbf{- 4}$ were thermally treated under the optimized conditions (sealed tube, $120{ }^{\circ} \mathrm{C}$ ). To our delight, pure compounds $\mathbf{5 a - l}$ were isolated in good to quantitative yields (60-100\%) by flash chromatography. Table 2 summarizes our results for the different $\beta$-lactams tested. 
Table 2. Synthesis of tetrahydroazocinones 5 through Cope reaction of E-cis-2-azetidinonetethered dienes $\mathbf{2}-\mathbf{4}^{a}$
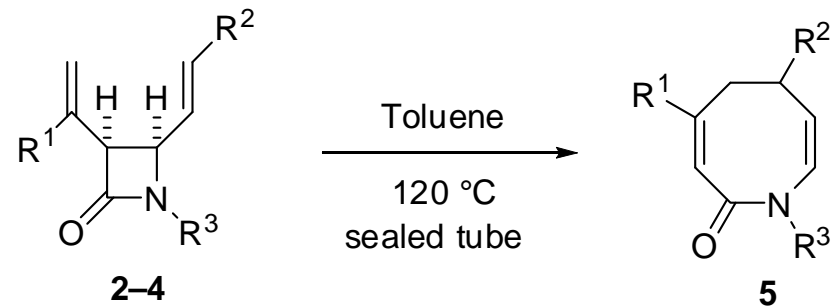

\begin{tabular}{|c|c|c|c|c|c|c|}
\hline (E)-Diene & $\mathrm{R}^{1}$ & $\mathrm{R}^{2}$ & $\mathrm{R}^{3}$ & Azocinone & $\mathrm{t}$ (h) & Yield (\%) \\
\hline$( \pm)-2 \mathbf{a}$ & $\mathrm{H}$ & $\mathrm{CO}_{2} \mathrm{Me}$ & PMP & $( \pm)-5 \mathbf{a}$ & 5 & 86 \\
\hline$( \pm)-2 \mathbf{b}$ & $\mathrm{Me}$ & $\mathrm{CO}_{2} \mathrm{Me}$ & PMP & $( \pm)-5 b$ & 5 & 90 \\
\hline$( \pm)-2 c$ & $\mathrm{H}$ & COMe & PMP & $( \pm)-5 c$ & 4 & 85 \\
\hline$( \pm)-2 d$ & $\mathrm{Me}$ & COMe & PMP & $( \pm)-5 d$ & 3.5 & 87 \\
\hline$( \pm)-2 e$ & $\mathrm{H}$ & $\mathrm{Ph}$ & PMP & $( \pm)-5 e$ & 5 & 70 \\
\hline$( \pm)-\mathbf{2} \mathbf{f}^{b}$ & $\mathrm{Me}$ & $\mathrm{Ph}$ & PMP & $( \pm)-5 f$ & 4 & $60^{c}$ \\
\hline$( \pm)-2 g$ & $\mathrm{H}$ & $\mathrm{CN}$ & PMP & $( \pm)-5 g$ & 2 & 100 \\
\hline$( \pm)-2 h$ & $\mathrm{Me}$ & $\mathrm{CN}$ & PMP & $( \pm)-5 h$ & 2 & 100 \\
\hline$( \pm)-3 a$ & $\mathrm{H}$ & $\mathrm{Ph}$ & $\mathrm{Bn}$ & $( \pm)-5 \mathbf{i}$ & 2.5 & 79 \\
\hline$( \pm)-3 \mathbf{b}$ & $\mathrm{Me}$ & $\mathrm{Ph}$ & $\mathrm{Bn}$ & $( \pm)-5 \mathbf{j}$ & 4 & 79 \\
\hline$(+)-4 a-\alpha$ & $\mathrm{H}$ & $\mathrm{Ph}$ & $(R)-\mathrm{CH}(\mathrm{Me}) \mathrm{Ph}$ & $(-)-5 \mathbf{k}-\alpha$ & 4 & 82 \\
\hline$(+)-4 a-\beta$ & $\mathrm{H}$ & $\mathrm{Ph}$ & $(R)-\mathrm{CH}(\mathrm{Me}) \mathrm{Ph}$ & $(+)-5 \mathbf{k}-\beta$ & 4 & 75 \\
\hline$(-)-4 b-\alpha$ & $\mathrm{Me}$ & $\mathrm{Ph}$ & $(R)-\mathrm{CH}(\mathrm{Me}) \mathrm{Ph}$ & $(-)-51-\alpha$ & 3 & 75 \\
\hline$(+)-4 b-\beta$ & $\mathrm{Me}$ & $\mathrm{Ph}$ & $(R)-\mathrm{CH}(\mathrm{Me}) \mathrm{Ph}$ & $(+)-51-\beta$ & 3 & 83 \\
\hline
\end{tabular}

${ }^{a} \mathrm{PMP}=4-\mathrm{MeOC}_{6} \mathrm{H}_{4} \cdot{ }^{b}$ Mixture of $\beta$-lactams $E$-cis/Z-cis/E-trans. ${ }^{c}$ Yield assuming that only the cis isomer reacts.

Replacing the $E$-isomer by the $Z$-isomer on the alkene moiety substituted by the $\mathrm{R}^{2}$ group in 2-azetidinone-tethered dienes 2 resulted in a remarkable loss of reactivity. When the $E$ configuration was switched to $Z$, dienes $\mathbf{2 g}$ and $\mathbf{2 h}$ were converted into the corresponding tetrahydroazocinones $\mathbf{5 g}$ and $\mathbf{5 h}$ in quantitative yields only under harsher conditions (sealed tube, $150{ }^{\circ} \mathrm{C}, 3-6 \mathrm{~h}$ ) (Scheme 4). The difference in reactivity of the $Z$ and $E$ isomers can be explained taking into account the higher steric and/or stereoelectronic interaction between the CN and PMP groups in the boat-like transition state for the $Z$ isomer in comparison with the $E$ isomer (Scheme 5). 


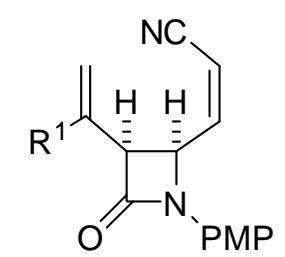

$Z-( \pm)-2 g: \mathrm{R}^{1}=\mathrm{H}$

$Z-( \pm)-2 h: R^{1}=M e$

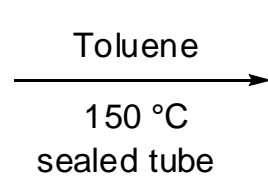

(quantitative yield)<smiles>[R]/C1=C/C(=O)N(P)/C=C/C(C#N)C1</smiles>

$( \pm)-5 g(6 h)$

$( \pm)-5 h$ (3 h)

\section{Scheme 4}

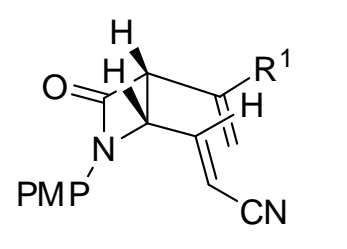

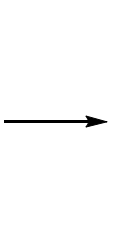

$E$ isomer<smiles></smiles>

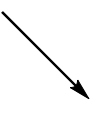<smiles>[R]/C1=C/C(=O)N(PN)C=CC(C#N)C1</smiles>

$5 g, 5 h$

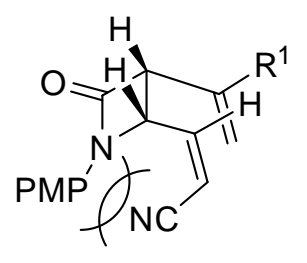
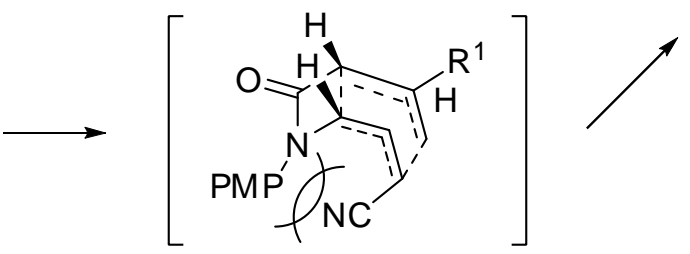

$Z$ isomer

\section{Scheme 5}

Of particular interest were the reactions of enantiomerically pure substrates $\mathbf{4 a}-\alpha, \beta$ and $\mathbf{4 b} \mathbf{b}$ $\alpha, \beta$ which cleanly rearranged to the corresponding optically pure tetrahydroazocinones $5 \mathbf{k}-\alpha, \beta$ and 5l- $\alpha, \beta$, respectively (Table 2). Also, when a mixture of isomers $(\alpha / \beta)$ of substrates $4 \mathbf{a}$ and $4 \mathbf{b}$ was used, a mixture of the corresponding azocinones $5 \mathbf{k}-\alpha, \beta$ and $5 \mathbf{l}-\alpha, \beta$ was obtained in the same relative proportions. Moreover, isomeric mixture $(\alpha / \beta)$ of compound $5 \mathbf{k}, \mathbf{l}$ was more amenable to chromatographic purification than the corresponding precursors, isomeric dienes 4a,b. Above results show, not unexpectedly, the stereospecificity of these Cope rearrangements (Scheme 6), which may be interpreted via a boat-like transition state. ${ }^{17}$ 


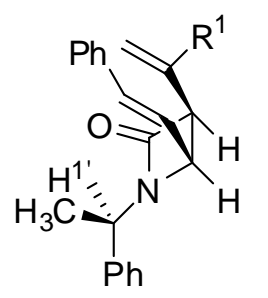

$4 \mathbf{a}-\alpha$

4b- $\alpha$

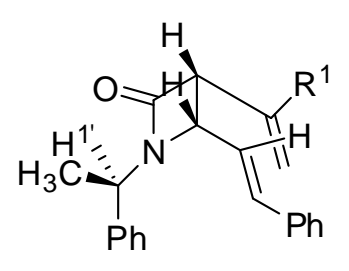

$4 a-\beta$

4b- $\beta$
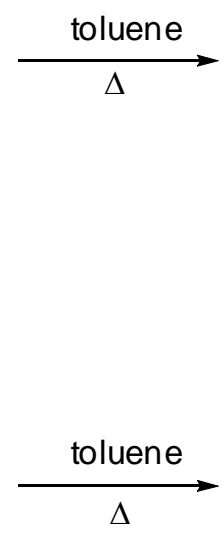

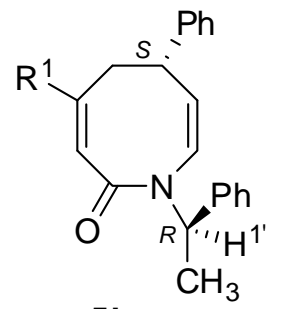

5k- $\alpha$

5l- $\alpha$

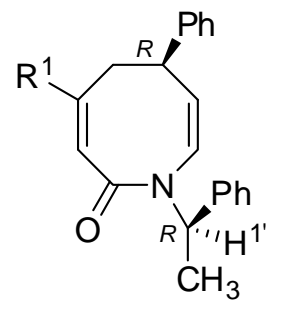

$5 k-\beta$

5I- $\beta$

\section{Scheme 6}

Replacing the cis- $\beta$-lactam by the trans- $\beta$-lactam isomer in 2-azetidinone-tethered dienes 2 resulted in a remarkable loss of efficiency. For dienes trans-2e and trans-3b, the rearrangement did not proceed under standard conditions. The desired [3,3] sigmatropic (Cope) rearrangement of trans- $\beta$-lactam diene trans-2e took place not very efficiently on heating in toluene at $180{ }^{\circ} \mathrm{C}$ in sealed tube, affording the corresponding azocinone 5e in a poor 30\% yield (Scheme 7). The conversion of diene trans-2e into azocinone 5e may involve an initial trans/cis isomerization through a diradical intermediate, followed by the Cope rearrangement of the in situ generated cis- $\beta$-lactam.
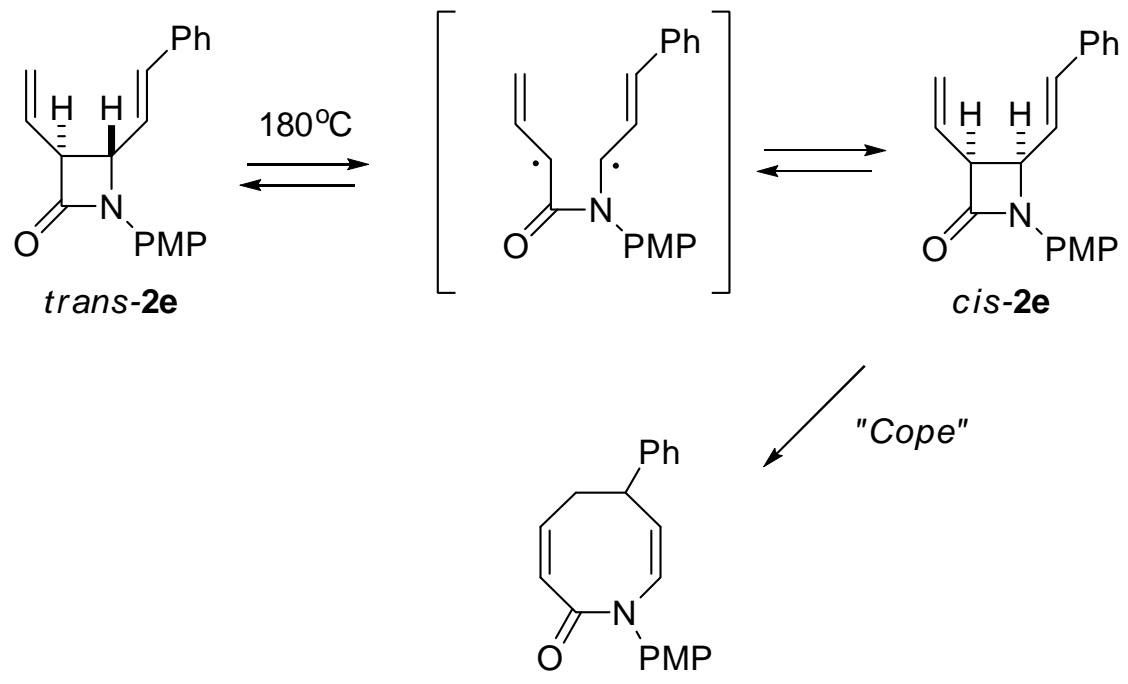

$( \pm)-5 e(30 \%)$

\section{Scheme 7}




\section{Conclusions}

The present study provides the first insight into the manner in which $c i s-\beta$-lactams having alk-2enyl groups both at the C3 and C4 positions undergo a thermally induced [3,3] sigmatropic (Cope) rearrangement to give new functionalized eight-membered lactam adducts (tetrahydroazocinones), in racemic as well as optically pure forms. This process involves a novel, concerted C3-C4 bond breakage of the $\beta$-lactam nucleus helped by ring strain.

\section{Experimental Section}

General Procedures. Melting points were measured by using a Gallen-kamp apparatus and are uncorrected. IR spectra were recorded with a Perkin-Elmer 781 spectrophotometer. ${ }^{1} \mathrm{H}$ NMR and ${ }^{13}$ C NMR spectra were recorded on a Bruker Avance-300, Varian VRX-300S or Bruker AC-200. NMR spectra were recorded in $\mathrm{CDCl}_{3}$ solutions, except otherwise stated. Chemical shifts are given in ppm relative to TMS $\left({ }^{1} \mathrm{H}, 0.0 \mathrm{ppm}\right)$, or $\mathrm{CDCl}_{3}\left({ }^{13} \mathrm{C}, 76.9 \mathrm{ppm}\right)$. Mass spectra were recorded with a HP5989A spectrometer using the electronic impact (EI) method. Optical rotations were measured by using a Perkin-Elmer 241 polarimeter. Specific rotation $[\alpha]_{\mathrm{D}}$ is given in $10^{-1}$ deg $\mathrm{cm}^{2} \mathrm{~g}^{-1}$ at $20{ }^{\circ} \mathrm{C}$, and the concentration $(c)$ is expressed in ger $100 \mathrm{~mL}$. Elemental analyses were obtained at the UCM Microanalysis Service (Facultad de Farmacia, UCM, 28040 Madrid). All commercially available compounds were used without further purification. Flash chromatography was performed by using Merck silica gel 60 (230-400 mesh). Products were identified by TLC (Kieselgel 60F-254). UV light $(\lambda=254 \mathrm{~nm})$ and a solution of phosphomolybdic acid in EtOH (1g of phosphomolybdic acid hydrate, $100 \mathrm{~mL}$ EtOH) were used to develop the plates.

\section{Preparation of dienes 2}

Method A. To a solution of the appropriate 4-oxoazetidine-2-carbaldehyde $\mathbf{1}(1.0 \mathrm{mmol})$ in anhydrous THF $(10 \mathrm{~mL})$, under argon atmosphere, the corresponding phosphorane $(1.40 \mathrm{mmol})$ was added portionwise and the mixture was heated at reflux temperature for $3 \mathrm{~h}$. The reaction mixture was allowed to cool to room temperature and the solvent was removed under reduced pressure. Chromatography of the residue using ethyl acetate/hexanes gave the corresponding dienes 2.

Method B. To a suspension of benzyltriphenylphosphonium bromide $(1.6 \mathrm{mmol})$ in anhydrous THF $(30 \mathrm{~mL})$, under argon atmosphere, $n \mathrm{BuLi}(1.6 \mathrm{M}$ in hexane, $1.40 \mathrm{mmol})$ was added dropwise. The red mixture was stirred for $20 \mathrm{~min}$ at room temperature and the appropriate 4oxoazetidine-2-carbaldehyde 1 (1 mmol) in THF $(10 \mathrm{~mL})$ was added. The reaction was stirred for $3 \mathrm{~h}$ and then was washed with $\mathrm{NaCl}$ (aq. sat.), the aqueous residue was extracted with AcOEt and the organic layer was dried $\left(\mathrm{MgSO}_{4}\right)$. The solvent was removed under reduced pressure. 
Chromatography of the residue eluting with hexanes/ethyl acetate gave analytically pure dienes 2.

Diene ( \pm -2a. Method A. From $100 \mathrm{mg}(0.43 \mathrm{mmol})$ of 4-oxoazetidine-2-carbaldehyde $( \pm)$-1a, $100 \mathrm{mg}(82 \%)$ of compound ( \pm )-2a was obtained as a white solid after purification by flash chromatography (hexanes/ethyl acetate, 4:1). Mp 116-118 ${ }^{\circ} \mathrm{C}$ (hexanes/ethyl acetate). NMR data: $\delta_{\mathrm{H}}\left(\mathrm{CDCl}_{3}\right) 3.68\left(\mathrm{~s}, 3 \mathrm{H}, \mathrm{OCH}_{3}\right), 3.72\left(\mathrm{~s}, 3 \mathrm{H}, \mathrm{OCH}_{3}\right), 4.16(\mathrm{t}, 1 \mathrm{H}, J=7.0 \mathrm{~Hz}, \mathrm{H} 3), 4.72$ (t, $1 \mathrm{H}, J=6.8 \mathrm{~Hz}, \mathrm{H} 4), 5.26-5.43\left(\mathrm{~m}, 2 \mathrm{H}, \mathrm{C}=\mathrm{CH}_{2}\right), 5.67$ (m, 1H, $\left.\mathrm{CH}=\mathrm{CH}_{2}\right), 6.01$ (d, $1 \mathrm{H}, J=15.8$ $\left.\mathrm{Hz}, \mathrm{CH}-\mathrm{CO}_{2} \mathrm{CH}_{3}\right), 6.83\left(\mathrm{~m}, 3 \mathrm{H}, \mathrm{CH}=\mathrm{CH}-\mathrm{CO}_{2} \mathrm{CH}_{3}, \mathrm{Ar}\right), 7.21(\mathrm{~m}, 2 \mathrm{H}, \mathrm{Ar}) . \delta_{\mathrm{C}}\left(\mathrm{CDCl}_{3}\right) 165.4$ (C=O), 163.8 (C2), 156.3 (Ar), 142.7, 130.8 (Ar), 127.9, $121.7\left(\mathrm{CH}=\mathrm{CH}_{2}\right), 118.1$ (Ar), 114.3 (Ar), $57.8(\mathrm{C} 4)$, $55.7\left(\mathrm{OCH}_{3}\right), 55.4\left(\mathrm{OCH}_{3}\right), 51.7(\mathrm{C} 3) . \mathrm{IR}\left(\mathrm{KBr}, \mathrm{cm}^{-1}\right): v 1735(\mathrm{NC}=\mathrm{O}), 1514$ $(\mathrm{C}=\mathrm{O})$. Anal. Calcd for $\mathrm{C}_{16} \mathrm{H}_{17} \mathrm{NO}_{4}$ : C, 66.89; H, 5.96; N, 4.88. Found: C, 66.75; H, 6.12; N, 4.99 .

Diene ( \pm -2b. Method A. From $302 \mathrm{mg}$ (1.30 mmol) of 4-oxoazetidine-2-carbaldehyde $( \pm)-1 \mathbf{b}$, $345 \mathrm{mg}(92 \%)$ of compound ( \pm )-2b was obtained as a white solid after purification by flash chromatography (hexanes/ethyl acetate, 5:1). Mp 123-125 ${ }^{\circ} \mathrm{C}$ (hexanes/ethyl acetate). NMR data: $\delta_{\mathrm{H}}\left(\mathrm{CDCl}_{3}\right) 1.62\left(\mathrm{~s}, 3 \mathrm{H}, \mathrm{CH}_{3}\right), 3.67\left(\mathrm{~s}, 3 \mathrm{H}, \mathrm{OCH}_{3}\right), 3.71\left(\mathrm{~s}, 3 \mathrm{H}, \mathrm{OCH}_{3}\right), 4.09(\mathrm{~d}, 1 \mathrm{H}, J=$ $6.0 \mathrm{~Hz}, \mathrm{H} 3$ ), 4.67 (t, 1H, $J=6.8 \mathrm{~Hz}, \mathrm{H} 4), 5.03$ (s, 1H, C=CHH), 5.12 (s, 1H, C=CHH), 6.04 (d, $\left.1 \mathrm{H}, J=15.8 \mathrm{~Hz}, \mathrm{CH}-\mathrm{CO}_{2} \mathrm{CH}_{3}\right), 6.80$ (m, 3H, $\left.\mathrm{CH}=\mathrm{CH}-\mathrm{CO}_{2} \mathrm{CH}_{3}, \mathrm{Ar}\right), 7.22$ (d, $2 \mathrm{H}, J=8.7 \mathrm{~Hz}$, $\mathrm{Ar}) . \delta_{\mathrm{C}}\left(\mathrm{CDCl}_{3}\right) 165.7(\mathrm{C}=\mathrm{O}), 164.0(\mathrm{C} 2), 156.4,142.5\left(\mathrm{C}=\mathrm{CH}_{2}\right), 135.9\left(\mathrm{CH}=\mathrm{CHCO}_{2} \mathrm{CH}_{3}\right)$, 131.1, $125.7\left(\mathrm{CH}=\mathrm{CHCO}_{2} \mathrm{CH}_{3}\right), 118.3,116.8\left(\mathrm{C}=\mathrm{CH}_{2}\right), 114.6,60.8,56.0\left(\mathrm{OCH}_{3}\right), 55.6$ $\left(\mathrm{OCH}_{3}\right)$, 52.0, $22.6\left(\mathrm{CH}_{3}\right)$. IR $\left(\mathrm{KBr}, \mathrm{cm}^{-1}\right): v 1735(\mathrm{NC}=\mathrm{O}), 1718(\mathrm{C}=\mathrm{O}), 1515$. Anal. Calcd for $\mathrm{C}_{17} \mathrm{H}_{19} \mathrm{NO}_{4}$ : C, 67.76; H, 6.36; N, 4.65. Found: C, 67.86; H, 6.22; N, 4.49.

Diene ( \pm )-2c. Method A. From $470 \mathrm{mg}$ (2.03 mmol) of 4-oxoazetidine-2-carbaldehyde $( \pm)$-1a, $375 \mathrm{mg}(68 \%)$ of compound $( \pm)$-2c was obtained as a white solid after purification by flash chromatography (hexanes/ethyl acetate 4:1). Mp 117-119 ${ }^{\circ} \mathrm{C}$ (hexanes/ethyl acetate). NMR data: $\delta_{\mathrm{H}}\left(\mathrm{CDCl}_{3}\right) 2.20\left(\mathrm{~s}, 3 \mathrm{H}, \mathrm{CH}_{3}\right), 3.71\left(\mathrm{~s}, 3 \mathrm{H}, \mathrm{OCH}_{3}\right), 4.17$ (dd, $\left.1 \mathrm{H}, J=6.2,7.4 \mathrm{~Hz}, \mathrm{H} 3\right), 4.72$ (dd, $1 \mathrm{H}, J=6.2,6.7 \mathrm{~Hz}, \mathrm{H} 4), 5.33\left(\mathrm{~m}, 2 \mathrm{H}, \mathrm{C}=\mathrm{CH}_{2}\right), 5.65\left(\mathrm{~m}, 1 \mathrm{H}, \mathrm{CH}=\mathrm{CH}_{2}\right), 6.24$ (d, $1 \mathrm{H}, J=16.2$ $\mathrm{Hz}, \mathrm{CH}-\mathrm{COCH}_{3}$ ), 6.66 (dd, $1 \mathrm{H} J=6.9,16.2 \mathrm{~Hz}, \mathrm{CH}=\mathrm{CH}-\mathrm{COCH}_{3}$ ), 6.80 (d, 2H, $J=8.7 \mathrm{~Hz}, \mathrm{Ar}$ ), $7.22(\mathrm{~m}, 2 \mathrm{H}, J=8.7 \mathrm{~Hz}, \mathrm{Ar}) . \delta_{\mathrm{C}}\left(\mathrm{CDCl}_{3}\right) 197.0(\mathrm{C}=\mathrm{O}), 163.8(\mathrm{C} 2), 156.4(\mathrm{Ar}), 141.3,134.0$, 131.0 (Ar), 128.0, $121.8\left(\mathrm{CH}=\mathrm{CH}_{2}\right), 118.1$ (Ar), 114.5 (Ar), 57.8, 56.0, 55.5, $27.6\left(\mathrm{CH}_{3}\right)$. IR $\left(\mathrm{KBr}, \mathrm{cm}^{-1}\right): v 1734(\mathrm{NC}=\mathrm{O}), 1670(\mathrm{C}=\mathrm{O}), 1518$. Anal. Calcd for $\mathrm{C}_{16} \mathrm{H}_{17} \mathrm{NO}_{3}$ : C, 70.83; H, 6.32; N, 5.16. Found: C, 70.88; H, 6.19; N, 5.23.

Diene $( \pm)$-2d. Method A. From 100mg (0.43 mmol) of 4-oxoazetidine-2-carbaldehyde $( \pm)-1 b$, $80 \mathrm{mg}(65 \%)$ of compound $( \pm)$-2d was obtained as a white solid after purification by flash chromatography (hexanes/ethyl acetate 4:1). Mp 116-118 ${ }^{\circ} \mathrm{C}$ (hexanes/ethyl acetate). NMR data: $\delta_{\mathrm{H}}\left(\mathrm{CDCl}_{3}\right) 1.61\left(\mathrm{~s}, 3 \mathrm{H}, \mathrm{CH}_{3}\right), 2.18\left(\mathrm{~s}, 3 \mathrm{H}, \mathrm{COCH}_{3}\right), 3.71\left(\mathrm{~s}, 3 \mathrm{H}, \mathrm{OCH}_{3}\right), 4.11(\mathrm{~d}, 1 \mathrm{H}, J=6.0$ $\mathrm{Hz}, \mathrm{H} 3), 4.67$ (t, 1H, $J=6.8 \mathrm{~Hz}, \mathrm{H} 4), 5.04(\mathrm{~s}, 1 \mathrm{H}, \mathrm{C}=\mathrm{CHH}), 5.14$ (s, 1H, C=CHH), 6.28 (d, 1H, $J=15.8 \mathrm{~Hz}, \mathrm{CH}-\mathrm{COCH}_{3}$ ), 6.64 (dd, $\left.1 \mathrm{H}, J=7.7,15.8 \mathrm{~Hz}, \mathrm{CH}=\mathrm{CH}-\mathrm{COCH}_{3}\right), 6.78$ (d, $2 \mathrm{H}, J=8.7$ $\mathrm{Hz}, \mathrm{Ar}), 7.25(\mathrm{~d}, 2 \mathrm{H}, J=8.7 \mathrm{~Hz}, \mathrm{Ar}) . \delta_{\mathrm{C}}\left(\mathrm{CDCl}_{3}\right) 197.1(\mathrm{C}=\mathrm{O}), 163.7(\mathrm{C} 2), 156.4,141.1$ $\left(C=\mathrm{CH}_{2}\right), 136.0\left(\mathrm{CH}_{\mathrm{CHCOCH}}\right), 134.6 \quad\left(\mathrm{CH}=\mathrm{CHCOCH}_{3}\right), 131.1,118.1,116.6\left(\mathrm{C}=\mathrm{CH}_{2}\right)$, 
114.5, 60.7 , 56.0 , $55.5\left(\mathrm{OCH}_{3}\right), 27.3\left(\mathrm{CH}_{3}\right), 22.4\left(\mathrm{CH}_{3}\right) . \mathrm{IR}\left(\mathrm{KBr}, \mathrm{cm}^{-1}\right): v 1732(\mathrm{NC}=\mathrm{O}), 1672$ $(\mathrm{C}=\mathrm{O}), 1639,1514$. Anal. Calcd for $\mathrm{C}_{17} \mathrm{H}_{19} \mathrm{NO}_{3}$ : C, 71.56; H, 6.71; N, 4.91. Found: C, 71.45; $\mathrm{H}$, $6.81 ; \mathrm{N}, 5.07$.

Diene ( \pm )-2e. Method B. From $100 \mathrm{mg}(0.43 \mathrm{mmol})$ of 4-oxoazetidine-2-carbaldehyde ( \pm )-1a, and after purification by flash chromatography (hexanes/ethyl acetate 4:1), $40 \mathrm{mg}$ (30\%) of $E$ cis-( \pm$)-2 \mathbf{e}$ and $60 \mathrm{mg}(46 \%)$ of E-trans-( \pm$)$-2e were obtained.

Diene E-cis-( \pm )-2e. Colourless oil. NMR data: $\delta_{\mathrm{H}}\left(\mathrm{CDCl}_{3}\right) 3.69\left(\mathrm{~s}, 3 \mathrm{H}, \mathrm{OCH}_{3}\right), 4.12$ (dd, $1 \mathrm{H}, J$ = 5.8, $7.4 \mathrm{~Hz}, \mathrm{H} 3), 4.72(\mathrm{dd}, 1 \mathrm{H}, J=5.8,8.2 \mathrm{~Hz}, \mathrm{H} 4), 5.31\left(\mathrm{~m}, 2 \mathrm{H}, \mathrm{C}=\mathrm{CH} \mathrm{H}_{2}\right), 5.76(\mathrm{~m}, 1 \mathrm{H}$, $\mathrm{CH}=\mathrm{CH}_{2}$ ), 6.15 (dd, $\left.1 \mathrm{H}, J=8.2,16.0 \mathrm{~Hz}, \mathrm{CH}=\mathrm{CHPh}\right), 6.76$ (m, 3H, $\left.\mathrm{CH}=\mathrm{CHPh}, \mathrm{Ar}\right), 7.31$ (m, 7H, Ar). $\delta_{\mathrm{C}}\left(\mathrm{CDCl}_{3}\right) 164.8(\mathrm{C} 2), 156.2(\mathrm{Ar}), 135.9,135.4,131.8,129.1,128.8,128.5,126.8$, 125.1, 121.0, 118.5, 114.4, 58.0 , 57.9, $55.6\left(\mathrm{OCH}_{3}\right)$. IR $\left(\mathrm{CHCl}_{3}, \mathrm{~cm}^{-1}\right): v 1740(\mathrm{NC}=\mathrm{O}), 1512$ $(\mathrm{C}=\mathrm{O})$. Anal. Calcd for $\mathrm{C}_{20} \mathrm{H}_{19} \mathrm{NO}_{2}$ : C, 78.66; H, 6.27; N, 4.59. Found: C, 78.55; H, 6.12; N, 4.42 .

Diene E-trans- $( \pm)$-2e. Colourless oil. NMR data: $\delta_{\mathrm{H}}\left(\mathrm{CDCl}_{3}\right) 3.68(\mathrm{~m}, 1 \mathrm{H}, \mathrm{H} 3), 3.69$ (s, 3H, $\mathrm{OCH}_{3}$ ), 4.35 (dd, 1H, $\left.J=2.3,8.4 \mathrm{~Hz}, \mathrm{H} 4\right), 5.27$ (s, 2H, C=CHH), 5.36 (s, 1H, C=CHH), 5.95 (m, $1 \mathrm{H}, \mathrm{CH}=\mathrm{CH}_{2}$ ), 6.22 (dd, $1 \mathrm{H}, J=8.4,16.0 \mathrm{~Hz}, \mathrm{CH}=\mathrm{CHPh}$ ), 6.70 (m, 3H, CH=CHPh, Ar), 7.13-7.35 (m, 7H, Ar). $\delta_{\mathrm{C}}\left(\mathrm{CDCl}_{3}\right) 164.3(\mathrm{C} 2), 156.0,135.8,134.9,131.6,129.3,128.6,128.5$, 127.9, 127.9, 119.8, 118.2, 114.2, 61.1 , 60.5, $55.6\left(\mathrm{OCH}_{3}\right) . \mathrm{IR}\left(\mathrm{CHCl}_{3}, \mathrm{~cm}^{-1}\right): v 1743(\mathrm{NC}=\mathrm{O})$, 1510 (C=O). Anal. Calcd for $\mathrm{C}_{20} \mathrm{H}_{19} \mathrm{NO}_{2}$ : C, 78.66; H, 6.27; N, 4.59. Found: C, 78.53; H, 6.00; $\mathrm{N}, 4.71$.

Diene $( \pm)$-2f. From $100 \mathrm{mg}(0.43 \mathrm{mmol})$ of 4-oxoazetidine-2-carbaldehyde $( \pm)-\mathbf{1 b}, 120 \mathrm{mg}$ (91\%) of an inseparable mixture of isomers E-cis/Z-cis/E-trans (45:50:15) were obtained as a colourless oil after purification by flash chromatography (hexanes/ethyl acetate 4:1). NMR data: $\delta_{\mathrm{H}}\left(\mathrm{CDCl}_{3}\right) 1.72\left(\mathrm{~s}, 3 \mathrm{H}, \mathrm{CH}_{3}\right), 1.84\left(\mathrm{~s}, 3 \mathrm{H}, \mathrm{CH}_{3}\right), 1.87\left(\mathrm{~s}, 3 \mathrm{H}, \mathrm{CH}_{3}\right.$ E-trans), 3.77 (s, 9H, $\mathrm{OCH}_{3}$ ), 3.84 (d, 1H, $J=2.5 \mathrm{~Hz}, \mathrm{H} 3$ E-trans), 4.13 (d, 1H, $J=6.1 \mathrm{~Hz}, \mathrm{H} 3 E$-cis), 4.20 (d, $1 \mathrm{H}, J=$ $5.9 \mathrm{~Hz}, \mathrm{H3} \mathrm{Z}$-cis), 4.47 (dd, 1H, $J=2.4,8.3 \mathrm{~Hz}, \mathrm{H} 4$ E-trans), 4.76 (dd, 1H, $J=5.9,8.8 \mathrm{~Hz}, \mathrm{H} 4$ E-cis), 5.01 (bs, 1H, C=CHH E-trans), 5.09-5.15 (m, 4H, $3 \mathrm{C}=\mathrm{CH}, \mathrm{H} 4 \mathrm{Z}-$ cis), 5.25 (bs, $2 \mathrm{H}, 2$ $\mathrm{C}=\mathrm{CH}$ ), 5.77 (dd, $1 \mathrm{H}, J=10.0,11.7 \mathrm{~Hz}, \mathrm{CH}=\mathrm{CHPh} \mathrm{Z}-c i s), 6.23$ (dd, $1 \mathrm{H}, J=8.8,15.9 \mathrm{~Hz}$, $\mathrm{CH}=\mathrm{CHPh}$ E-cis), 6.33 (dd, $1 \mathrm{H}, J=8.3,15.9 \mathrm{~Hz}, \mathrm{CH}=\mathrm{CHPh}$ E-trans), 6.75-6.90 (m, 9H, $\mathrm{CH}=\mathrm{CHPh}, \mathrm{PMP})$, 7.20-7.50 (m, 21H, Ph, PMP).

Diene $( \pm)$-2g. From $94 \mathrm{mg}(0.41 \mathrm{mmol})$ of 4-oxoazetidine-2-carbaldehyde $( \pm)$-1a, and after purification by flash chromatography (hexanes/ethyl acetate 5:1), $36 \mathrm{mg}$ (35\%) of Z-cis-( \pm )-2g and $57 \mathrm{mg}$ (55\%) of E-cis-( \pm )-2g were obtained.

Diene Z-cis- $( \pm)$-2g. White solid. Mp $125-127{ }^{\circ} \mathrm{C}$ (hexanes/ethyl acetate). NMR data: $\delta_{\mathrm{H}}$ $\left(\mathrm{CDCl}_{3}\right) 3.79$ (s, 3H, $\mathrm{OCH}_{3}$ ), 4.35 (bdd, $1 \mathrm{H}, J=6.0,7.3 \mathrm{~Hz}, \mathrm{H3}$ ), 5.11 (ddd, $1 \mathrm{H}, J=0.8$, 5.9, 9.7 $\mathrm{Hz}, \mathrm{H} 4$ ), 5.40 (dd, $1 \mathrm{H}, J=1.2,10.3 \mathrm{~Hz}, \mathrm{CH}=\mathrm{CHH}$ ), 5.54 (dd, $1 \mathrm{H}, J=1.3,17.0 \mathrm{~Hz}, \mathrm{CH}=\mathrm{CH} H$ ), 5.72 (dd, $1 \mathrm{H}, J=1.0,11.0 \mathrm{~Hz}, \mathrm{CH}=\mathrm{CN}$ ), 5.75 (ddd, $1 \mathrm{H}, J=7.3,10.3,17.2 \mathrm{~Hz}, \mathrm{CH}=\mathrm{CH}_{2}$ ), 6.54 (dd, $1 \mathrm{H} J=9.7,11.0 \mathrm{~Hz}, \mathrm{CH}=\mathrm{CH}-\mathrm{CN}), 6.88(\mathrm{~m}, 2 \mathrm{H}, \mathrm{Ar}), 7.28(\mathrm{~m}, 2 \mathrm{H}, \mathrm{Ar}) . \delta_{\mathrm{C}}\left(\mathrm{CDCl}_{3}\right) 163.4$ (C2), 156.5, $150.4(\mathrm{CH}=\mathrm{CH}-\mathrm{CN}), 130.7,127.2\left(\mathrm{CH}=\mathrm{CH}_{2}\right), 122.2\left(=\mathrm{CH}_{2}\right), 118.0,114.6(\mathrm{CN})$, 114.5, $104.8(\mathrm{CH}=\mathrm{CH}-\mathrm{CN}), 57.5(\mathrm{C} 3), 55.4\left(\mathrm{OCH}_{3}\right), 54.8(\mathrm{C} 4) . \mathrm{IR}\left(\mathrm{KBr}, \mathrm{cm}^{-1}\right): v 1740$ 
$(\mathrm{NC}=\mathrm{O})$. Anal. Calcd for $\mathrm{C}_{15} \mathrm{H}_{14} \mathrm{~N}_{2} \mathrm{O}_{2}$ : C, 70.85; H, 5.55; N, 11.02. Found: C, 70.88; H, 5.69; N, 10.98 .

Diene $\boldsymbol{E}$-cis- $( \pm)$-2g. Colourless oil. NMR data: $\delta_{\mathrm{H}}\left(\mathrm{CDCl}_{3}\right) 3.80\left(\mathrm{~s}, 3 \mathrm{H}, \mathrm{OCH}_{3}\right), 4.26$ (dd, $1 \mathrm{H}, J$ = 6.6, $7.1 \mathrm{~Hz}, \mathrm{H3}$ ), 4.78 (dt, $1 \mathrm{H}, J=0.8,6.6 \mathrm{~Hz}, \mathrm{H} 4), 5.42$ (d, $1 \mathrm{H}, J=10.1 \mathrm{~Hz}, \mathrm{CH}=\mathrm{CHH}), 5.49$ (d, $1 \mathrm{H}, J=17.0 \mathrm{~Hz}, \mathrm{CH}=\mathrm{CH} H$ ), 5.60 (dd, $1 \mathrm{H}, J=0.8,16.5 \mathrm{~Hz}, \mathrm{CH}=\mathrm{CN}$ ), 5.72 (ddd, $1 \mathrm{H}, J=7.8$, 10.1, 17.3 Hz, $\mathrm{CH}=\mathrm{CH}_{2}$ ), 6.71 (dd, $1 \mathrm{H} J=6.4,16.5 \mathrm{~Hz}, \mathrm{CH}=\mathrm{CH}-\mathrm{CN}$ ), 6.88 (m, 2H, Ar), 7.26 (m, 2H, Ar). $\delta_{\mathrm{C}}\left(\mathrm{CDCl}_{3}\right) 163.4(\mathrm{C} 2), 156.6,149.3(\mathrm{CH}=\mathrm{CH}-\mathrm{CN}), 130.4,127.4\left(\mathrm{CH}=\mathrm{CH}_{2}\right), 122.6$ $\left(=\mathrm{CH}_{2}\right), 118.1,115.9(\mathrm{CN}), 114.6,104.2(\mathrm{CH}=\mathrm{CH}-\mathrm{CN}), 58.0(\mathrm{C} 3), 55.9(\mathrm{C} 4), 55.5\left(\mathrm{OCH}_{3}\right) . \mathrm{IR}$ $\left(\mathrm{CHCl}_{3}, \mathrm{~cm}^{-1}\right): v 1743(\mathrm{NC}=\mathrm{O})$. MS (EI), m/z: $255\left(\mathrm{M}^{+}+1,7\right), 254\left(\mathrm{M}^{+}, 38\right), 171\left(\mathrm{M}^{+}-83,30\right)$, 149 (100).

Diene $( \pm)$-2h. From $137 \mathrm{mg}(0.59 \mathrm{mmol})$ of 4-oxoazetidine-2-carbaldehyde $( \pm)$-1b, and after purification by flash chromatography (hexanes/ethyl acetate 3:1), $90 \mathrm{mg}$ (57\%) of Z-( \pm )-2h and $53 \mathrm{mg}$ (55\%) of $E-( \pm)$-2h were obtained.

Diene Z-( \pm )-2h. White solid. Mp 137-139 ${ }^{\circ} \mathrm{C}$ (hexanes/ethyl acetate). NMR data: $\delta_{\mathrm{H}}\left(\mathrm{CDCl}_{3}\right)$ 1.72 (s, 3H, $\mathrm{CH}_{3}$ ), 3.79 (s, 3H, $\mathrm{OCH}_{3}$ ), 4.26 (d, 1H, $J=5.9 \mathrm{~Hz}, \mathrm{H} 3$ ), 5.10 (ddd, $1 \mathrm{H}, J=0.8,5.9$, $9.9 \mathrm{~Hz}, \mathrm{H} 4), 5.14$ (s, 1H, C=CHH), 5.26 (d, 1H, $J=1.0 \mathrm{~Hz}, \mathrm{C}=\mathrm{CH} H), 5.71$ (dd, $1 \mathrm{H}, J=0.8,11.0$ $\mathrm{Hz}, \mathrm{CH}=\mathrm{CN}$ ), 6.54 (dd, $1 \mathrm{H} J=9.9,11.0 \mathrm{~Hz}, \mathrm{CH}=\mathrm{CH}-\mathrm{CN}), 6.88$ (m, 2H, Ar), 7.30 (m, 2H, Ar). $\delta_{\mathrm{C}}\left(\mathrm{CDCl}_{3}\right) 163.4(\mathrm{C} 2), 156.6,150.4(\mathrm{CH}=\mathrm{CH}-\mathrm{CN}), 135.6\left(C=\mathrm{CH}_{2}\right), 130.8,118.1,116.7$ (= $\left.=\mathrm{CH}_{2}\right), 114.6,114.5(\mathrm{CN}), 104.9(\mathrm{CH}=\mathrm{CH}-\mathrm{CN}), 60.4(\mathrm{C}), 55.5\left(\mathrm{OCH}_{3}\right), 54.6(\mathrm{C} 4), 22.3$ $\left(\mathrm{CH}_{3}\right)$. IR $\left(\mathrm{KBr}, \mathrm{cm}^{-1}\right): v 1742(\mathrm{NC}=\mathrm{O})$. MS (EI), m/z: $269\left(\mathrm{M}^{+}+1,6\right), 268\left(\mathrm{M}^{+}, 29\right), 186\left(\mathrm{M}^{+}-\right.$ 82, 44), 149 (100). Anal. Calcd for $\mathrm{C}_{16} \mathrm{H}_{16} \mathrm{~N}_{2} \mathrm{O}_{2}$ : C, 71.62; H, 6.01; N, 10.44. Found: C, 71.70; H, 5.99; N, 10.48 .

Diene $\boldsymbol{E}-( \pm)-2 \mathbf{h}$. White solid. Mp $112-114{ }^{\circ} \mathrm{C}$ (hexanes/ethyl acetate). NMR data: $\delta_{\mathrm{H}}\left(\mathrm{CDCl}_{3}\right)$. NMR data: $\delta_{\mathrm{H}}\left(\mathrm{CDCl}_{3}\right) 1.70\left(\mathrm{~s}, 3 \mathrm{H}, \mathrm{CH}_{3}\right), 3.79\left(\mathrm{~s}, 3 \mathrm{H}, \mathrm{OCH}_{3}\right), 4.19$ (d, $1 \mathrm{H}, J=6.0 \mathrm{~Hz}, \mathrm{H} 3$ ), $4.73(\mathrm{td}, 1 \mathrm{H}, J=1.0,6.6 \mathrm{~Hz}, \mathrm{H} 4), 5.15(\mathrm{~s}, 1 \mathrm{H}, \mathrm{C}=\mathrm{CHH}), 5.19(\mathrm{~d}, 1 \mathrm{H}, J=0.9 \mathrm{~Hz}, \mathrm{C}=\mathrm{CH} H), 5.64$ (dd, $1 \mathrm{H}, J=1.0,16.4 \mathrm{~Hz}, \mathrm{CH}=\mathrm{CN}$ ), 6.70 (dd, $1 \mathrm{H} J=7.0,16.4 \mathrm{~Hz}, \mathrm{CH}=\mathrm{CH}-\mathrm{CN}$ ), 6.87 (m, 2H, $\mathrm{Ar}), 7.26(\mathrm{~m}, 2 \mathrm{H}, \mathrm{Ar}) . \delta_{\mathrm{C}}\left(\mathrm{CDCl}_{3}\right) 163.3(\mathrm{NC}=\mathrm{O}), 156.6,148.8(\mathrm{CH}=\mathrm{CH}-\mathrm{CN}), 135.3\left(C=\mathrm{CH}_{2}\right)$, 130.5, 118.1, 117.2 (= $\left.=\mathrm{CH}_{2}\right), 115.9(\mathrm{CN}), 114.5,104.5$ (CH=CH-CN), 61.0 (C3), 55.9 (C4), 55.5 $\left(\mathrm{OCH}_{3}\right), 22.4(\mathrm{Me})$. IR $\left(\mathrm{KBr}, \mathrm{cm}^{-1}\right): v 1743(\mathrm{NC}=\mathrm{O})$. Anal. Calcd for $\mathrm{C}_{16} \mathrm{H}_{16} \mathrm{~N}_{2} \mathrm{O}_{2}$ : C, 71.62; $\mathrm{H}$, 6.01; N, 10.44. Found: C, 71.65; H, 6.10; N, 10.50.

Preparation of dienes 3 . A suspension of benzylamine (10 mmol), cynnamaldehyde (10 mmol) and magnesium sulphate $(80 \mathrm{mmol})$ in dichloromethane $(100 \mathrm{~mL})$ was stirred at room temperature for $12 \mathrm{~h}$. The resulting imine was filtered and the solvent was removed under reduced pressure. The corresponding imine was dissolved in dichloromethane $(100 \mathrm{~mL})$ and $\mathrm{Et}_{3} \mathrm{~N}$ (20 mmol) was added dropwise followed by the corresponding acid chloride (12 mmol) and the resulting mixture was refluxed for $5 \mathrm{~h}$. The reaction was allowed to cool to room temperature and the crude mixture was washed with water $(50 \mathrm{~mL})$ and the aqueous layer was extracted with dichloromethane. The organic layer was dried $\left(\mathrm{MgSO}_{4}\right)$ and concentrated under reduced pressure. Chromatography of the residue eluting with hexanes/ethyl acetate gave analytically pure compounds 3 . 
Diene cis-( \pm )-3a. From $1.07 \mathrm{~g} \mathrm{(10} \mathrm{mmol)} \mathrm{of} \mathrm{benzylamine,} 1.32 \mathrm{~g}$ of cynnamaldehyde (10 mmol) and $1.14 \mathrm{~mL}(12 \mathrm{mmol})$ of crotonyl chloride, $1.60 \mathrm{~g}(56 \%)$ of compound $( \pm)$-3a was obtained as a colourless oil after purification by flash chromatography (hexanes/ethyl acetate 4:1) NMR data: $\delta_{\mathrm{H}}\left(\mathrm{CDCl}_{3}\right) 4.08(\mathrm{~m}, 2 \mathrm{H}, \mathrm{H} 3, \mathrm{NCHH}), 4.23(\mathrm{dd}, 1 \mathrm{H}, J=5.6,8.8 \mathrm{~Hz}, \mathrm{H} 4), 4.69$ (d, 1H, J = 14.9 $\mathrm{Hz}, \mathrm{NCH} H$ ), 5.25-5.46 (m, 2H, C=C $\mathrm{CH}_{2}$ ), $5.81\left(\mathrm{~m}, 1 \mathrm{H}, \mathrm{CH}=\mathrm{CH}_{2}\right), 6.02(\mathrm{dd}, 1 \mathrm{H}, J=8.8,15.9 \mathrm{~Hz}$, $\mathrm{CH}=\mathrm{CHPh}), 6.53(\mathrm{~d}, 1 \mathrm{H}, J=15.9 \mathrm{~Hz}, \mathrm{CH}=\mathrm{C} H \mathrm{Ph}), 7.25(\mathrm{~m}, 10 \mathrm{H}, \mathrm{Ar}) . \delta_{\mathrm{C}}\left(\mathrm{CDCl}_{3}\right) 167.6(\mathrm{C} 2)$, 135.9, 135.8, 135.5, 129.2, 128.8, 128.6, 128.5, 128.2, 127.7, 126.6, 124.7, $120.3\left(\mathrm{CH}=\mathrm{CH}_{2}\right)$, 58.1 , 57.7, $44.6\left(\mathrm{NCH}_{2}\right)$. IR $\left(\mathrm{CHCl}_{3}, \mathrm{~cm}^{-1}\right): v 1743(\mathrm{NC}=\mathrm{O})$. Anal. Calcd for $\mathrm{C}_{20} \mathrm{H}_{19} \mathrm{NO}: \mathrm{C}$, 83.01; H, 6.62; N, 4.84. Found: C, 80.18; H, 6.54; N, 5.03.

Diene ( \pm )-3b. From $353 \mathrm{mg}$ (3.3 mmol) of benzylamine, $397 \mathrm{mg}$ (3.3 mmol) of cynnamaldehyde and $0.44 \mathrm{~mL}$ (4 mmol) of 3,3-dimethylacryloyl chloride, $540 \mathrm{mg}(54 \%)$ of isomer cis- ( \pm )-3b and $30 \mathrm{mg}(3 \%)$ of isomer trans-( \pm -3b were obtained, after purification by flash chromatography (hexanes/ethyl acetate 4:1).

Diene cis- $( \pm)$-3b. Colourless oil. NMR data: $\delta_{\mathrm{H}}\left(\mathrm{CDCl}_{3}\right) 1.56$ (s, 3H, $\left.\mathrm{CH}_{3}\right), 3.89(\mathrm{~d}, 1 \mathrm{H}, J=5.4$ Hz, H3), 3.99 (d, 1H, $J=15.2 \mathrm{~Hz}, \mathrm{NCHH}$ ), 4.11 (dd, 1H, $J=5.4,9.3 \mathrm{~Hz}, \mathrm{H} 4), 4.59$ (d, $1 \mathrm{H}, J=$ $15.2 \mathrm{~Hz}, \mathrm{NCH} H$ ), 4.98 (s, $1 \mathrm{H}, \mathrm{C}=\mathrm{CHH}$ ), 5.12 (s, $1 \mathrm{H}, \mathrm{C}=\mathrm{CH} H$ ), 5.91 (dd, $1 \mathrm{H}, J=9.3,15.6 \mathrm{~Hz}$, $\mathrm{C} H=\mathrm{CHPh}), 6.46(\mathrm{~d}, 1 \mathrm{H}, J=15.6 \mathrm{~Hz}, \mathrm{CH}=\mathrm{C} H \mathrm{Ph}), 7.22(\mathrm{~m}, 10 \mathrm{H}, \mathrm{Ar}) . \delta_{\mathrm{C}}\left(\mathrm{CDCl}_{3}\right) 167.6(\mathrm{C} 2)$, 137.4, 136.2, 136.1, 135.9, 128.9, 128.8, 128.6, 128.3, 127.8, 126.7, 124.5, $115.0\left(\mathrm{C}=\mathrm{CH}_{2}\right), 60.9$ (C3), 57.7 (C4), $44.5\left(\mathrm{NCH}_{2}\right), 22.5\left(\mathrm{CH}_{3}\right)$. IR $\left(\mathrm{CHCl}_{3}, \mathrm{~cm}^{-1}\right): v 1749(\mathrm{NC}=\mathrm{O})$. Anal. Calcd for $\mathrm{C}_{21} \mathrm{H}_{21} \mathrm{NO}$ : C, 83.13; H, 6.98; N, 4.62. Found: C, 82.95; H, 7.18; N, 4.88.

Diene trans- $( \pm)-3 \mathrm{~b}$. Colourless oil. NMR data: $\delta_{\mathrm{H}}\left(\mathrm{CDCl}_{3}\right) 1.76\left(\mathrm{~s}, 3 \mathrm{H}, \mathrm{CH}_{3}\right), 3.63$ (bs, $\left.1 \mathrm{H}, \mathrm{H} 3\right)$, 3.90 (dd, $1 \mathrm{H}, J=2.2,8.7 \mathrm{~Hz}, \mathrm{H} 4), 4.03$ (d, $1 \mathrm{H}, J=15.0 \mathrm{~Hz}, \mathrm{NCHH}), 4.74$ (d, $1 \mathrm{H}, J=15.0 \mathrm{~Hz}$, $\mathrm{NCH} H), 4.93(\mathrm{~s}, 1 \mathrm{H}, \mathrm{C}=\mathrm{C} H \mathrm{H}), 5.00(\mathrm{~s}, 1 \mathrm{H}, \mathrm{C}=\mathrm{CH} H), 6.10(\mathrm{dd}, 1 \mathrm{H}, J=8.7,15.8 \mathrm{~Hz}$, $\mathrm{CH}=\mathrm{CHPh}$ ), 6.55 (d, $1 \mathrm{H}, J=15.8 \mathrm{~Hz}, \mathrm{CH}=\mathrm{CHPh}), 7.31(\mathrm{~m}, 10 \mathrm{H}, \mathrm{Ar}) . \delta_{\mathrm{C}}\left(\mathrm{CDCl}_{3}\right) 167.5(\mathrm{C} 2)$, 138.5, 136.1, 136.0, 134.7, 128.9, 128.9, 128.7, 128.5, 127.9, 126.8, 126.7, $114.3\left(\mathrm{C}=\mathrm{CH}_{2}\right), 64.6$ (C3), 59.2 (C4), $44.8\left(\mathrm{NCH}_{2}\right), 20.5\left(\mathrm{CH}_{3}\right)$. IR $\left(\mathrm{CHCl}_{3}, \mathrm{~cm}^{-1}\right): v 1747(\mathrm{NC}=\mathrm{O})$. Anal. Calcd for $\mathrm{C}_{21} \mathrm{H}_{21} \mathrm{NO}$ : C, 83.13; H, 6.98; N, 4.62. Found: C, 82.99; H, 7.12; N, 4.85.

Preparation of dienes 4 . A mixture of $(R)$-1-phenylethylamine (10 mmol), cynnammaldehyde (10 mol) and crotonyl chloride (for 4a) or 3,3-dimethylacryloyl (for $4 \mathbf{b}$ ) in anhydrous dichloromethane $(100 \mathrm{~mL})$ was stirred for $5 \mathrm{~h}$ at room temperature. The crude mixture was washed with water $(50 \mathrm{~mL})$ and the aqueous layer was extracted with dichloromethane. The organic layer was dried $\left(\mathrm{MgSO}_{4}\right)$ and concentrated under reduced pressure. Chromatography of the residue eluting with hexanes/ethyl acetate (5:1) gave analytically pure $1.85 \mathrm{~g}$ (61\%) of compound $\mathbf{4 a}$ as a mixture of diastereomers (56:44) and $1.80 \mathrm{~g}(57 \%)$ of compound $\mathbf{4 b}$, as a mixture of diastereomers (64:36). Diastereomers were separated after a second flash chromatography using hexanes/ethyl acetate (3:1).

Diene (+)-4a- $\alpha$. Colourless oil (34\%). $[\alpha]_{\mathrm{D}}=+95.0\left(c 1.0, \mathrm{CHCl}_{3}\right)$. NMR data: $\delta_{\mathrm{H}}\left(\mathrm{CDCl}_{3}\right) 1.51$ (d, 3H, $J=7.2 \mathrm{~Hz}, \mathrm{CH}_{3}$ ), 3.84 (dd, $1 \mathrm{H}, J=5.8,7.2 \mathrm{~Hz}, \mathrm{H} 3$ ), 4.05 (m, 1H, H4), 4.92 (q, $1 \mathrm{H}, J$ $=7.2 \mathrm{~Hz}, \mathrm{NCH}), 5.27\left(\mathrm{~m}, 2 \mathrm{H}, \mathrm{C}=\mathrm{CH}_{2}\right), 5.70\left(\mathrm{~m}, 1 \mathrm{H}, \mathrm{CH}=\mathrm{CH}_{2}\right), 6.03(\mathrm{dd}, 1 \mathrm{H}, J=6.5,15.8 \mathrm{~Hz}$, $\mathrm{CH}=\mathrm{CHPh}), 6.37$ (d, $1 \mathrm{H}, J=15.8 \mathrm{~Hz}, \mathrm{CH}=\mathrm{C} H \mathrm{Ph}), 7.22(\mathrm{~m}, 10 \mathrm{H}, \mathrm{Ar}) . \delta_{\mathrm{C}}\left(\mathrm{CDCl}_{3}\right) 167.2(\mathrm{C} 2)$, 
140.2, 136.0, 134.4, 129.4, 128.6, 128.6, 128.2, 127.7, 127.3, 126.7, 126.6, $120.2\left(\mathrm{CH}=\mathrm{CH}_{2}\right)$, 57.6, 57.4, $51.9(\mathrm{NCH}), 19.5\left(\mathrm{CH}_{3}\right)$. IR $\left(\mathrm{CHCl}_{3}, \mathrm{~cm}^{-1}\right): v 1740(\mathrm{NC}=\mathrm{O})$. Anal. Calcd for $\mathrm{C}_{21} \mathrm{H}_{21} \mathrm{NO}$ : C, 83.13; H, 6.98; N, 4.62. Found: C, 83.24; H, 7.11; N, 4.51.

Diene (+)-4a- $\beta$. Colourless oil (27\%). $[\alpha]_{\mathrm{D}}=+325.0\left(c\right.$ 1.0, $\left.\mathrm{CHCl}_{3}\right)$. NMR data: $\delta_{\mathrm{H}}\left(\mathrm{CDCl}_{3}\right)$ 1.65 (d, $3 \mathrm{H}, J=7.2 \mathrm{~Hz}, \mathrm{CH}_{3}$ ), 3.90 (dd, $1 \mathrm{H}, J=5.6,7.2 \mathrm{~Hz}, \mathrm{H} 3$ ), 4.18 (dd, $1 \mathrm{H}, J=5.6,9.1 \mathrm{~Hz}$, $\mathrm{H} 4), 4.61$ (q, $1 \mathrm{H}, J=7.2 \mathrm{~Hz}, \mathrm{NCH}), 5.22\left(\mathrm{~m}, 2 \mathrm{H}, \mathrm{CH}=\mathrm{CH}_{2}\right), 5.60-5.77\left(\mathrm{~m}, 2 \mathrm{H}, \mathrm{CH}=\mathrm{CH}_{2}\right.$, $\mathrm{CH}=\mathrm{CHPh}), 6.37$ (d, $1 \mathrm{H}, J=15.8 \mathrm{~Hz}, \mathrm{CH}=\mathrm{C} H \mathrm{Ph}), 7.20(\mathrm{~m}, 10 \mathrm{H}, \mathrm{Ar}) . \delta_{\mathrm{C}}\left(\mathrm{CDCl}_{3}\right) 167.2(\mathrm{C} 2)$, 141.3, 135.8, 134.7, 129.2, 128.4, 128.4, 128.0, 127.4, 126.8, 126.4, 125.4, $120.0\left(\mathrm{CH}=\mathrm{CH}_{2}\right)$, 57.1, 57.0, $52.7(\mathrm{NCH}), 18.7\left(\mathrm{CH}_{3}\right)$. IR $\left(\mathrm{CHCl}_{3}, \mathrm{~cm}^{-1}\right): v 1739$ (NC=O), 1494. MS (EI), $m / z: 304$ $\left(\mathrm{M}^{+}+1,2\right), 303\left(\mathrm{M}^{+}, 6\right), 199\left(\mathrm{M}^{+}-104,86\right), 105$ (100).Anal. Calcd for $\mathrm{C}_{21} \mathrm{H}_{21} \mathrm{NO}$ : C, 83.13; $\mathrm{H}$, 6.98; N, 4.62. Found: C, 83.26; H, 7.05; N, 4.45.

Diene (-)-4b- $\alpha$. Colourless oil (37\%). $[\alpha]_{\mathrm{D}}=-117.0\left(c\right.$ 1.1, $\left.\mathrm{CHCl}_{3}\right)$. NMR data: $\delta_{\mathrm{H}}\left(\mathrm{CDCl}_{3}\right)$ 1.59 (d, 3H, $J=7.4 \mathrm{~Hz}, \mathrm{CHPh}-\mathrm{CH}_{3}$ ), 1.61 (s, 3H, $\mathrm{CH}_{3}$ ), 3.84 (d, $1 \mathrm{H}, J=5.6 \mathrm{~Hz}, \mathrm{H} 3$ ), 4.10 (dd, $1 \mathrm{H}, J=5.6,9.4 \mathrm{~Hz}, \mathrm{H} 4), 5.04$ (q, $1 \mathrm{H}, J=7.0 \mathrm{~Hz}, \mathrm{NCH}$ ), 5.05 (s, $1 \mathrm{H}, \mathrm{C}=\mathrm{C} H \mathrm{H}), 5.19$ (s, $1 \mathrm{H}$, $\mathrm{C}=\mathrm{CH} H$ ), 6.08 (dd, $1 \mathrm{H}, J=9.4,15.8 \mathrm{~Hz}, \mathrm{CH}=\mathrm{CHPh}), 6.47$ (d, $1 \mathrm{H}, J=15.8 \mathrm{~Hz}, \mathrm{CH}=\mathrm{CHPh}$ ), $7.32(\mathrm{~m}, 10 \mathrm{H}, \mathrm{Ar}) . \delta_{\mathrm{C}}\left(\mathrm{CDCl}_{3}\right) 167.0(\mathrm{C} 2), 140.0,137.3,136.1,134.5,128.6,128.3,128.0$, 127.6, 127.2, 126.5, 126.3, $114.7\left(\mathrm{C}=\mathrm{CH}_{2}\right), 59.9,57.3,51.6(\mathrm{NCH}), 22.3\left(\mathrm{CH}_{3}\right), 19.5\left(\mathrm{CH}_{3}\right) . \mathrm{IR}$ $\left(\mathrm{CHCl}_{3}, \mathrm{~cm}^{-1}\right)$ : v $1750(\mathrm{NC}=\mathrm{O})$. Anal. Calcd for $\mathrm{C}_{21} \mathrm{H}_{21} \mathrm{NO}$ : C, 83.24; H, 7.30; N, 4.41. Found: C, 83.37; H, 7.17; N, 4.61.

Diene (+)-4b- $\beta$. Colourless oil (20\%). $[\alpha]_{\mathrm{D}}=+85.0\left(c 0.8, \mathrm{CHCl}_{3}\right)$. NMR data: $\delta_{\mathrm{H}}\left(\mathrm{CDCl}_{3}\right) 1.53$ (s, 3H, $\mathrm{CH}_{3}$ ), 1.65 (d, 3H, $J=7.2 \mathrm{~Hz}, \mathrm{CHPh}-\mathrm{CH}_{3}$ ), 3.81 (d, $1 \mathrm{H}, J=5.6 \mathrm{~Hz}, \mathrm{H} 3$ ), 4.14 (dd, $1 \mathrm{H}, J$ = 5.6, $9.4 \mathrm{~Hz}, \mathrm{H} 4), 4.60$ (c, $1 \mathrm{H}, J=7.2 \mathrm{~Hz}, \mathrm{NCH}), 5.10$ (s, $1 \mathrm{H}, \mathrm{C}=\mathrm{C} H \mathrm{H}), 5.22(\mathrm{~s}, 1 \mathrm{H}, \mathrm{C}=\mathrm{CH} H$ ), 5.76 (dd, $1 \mathrm{H}, J=9.4,15.9 \mathrm{~Hz}, \mathrm{CH}=\mathrm{CHPh}$ ), 6.40 (d, $1 \mathrm{H}, J=15.9 \mathrm{~Hz}, \mathrm{CH}=\mathrm{C} H \mathrm{Ph}$ ), 7.25 (m, 10H, $\mathrm{Ar}) . \delta_{\mathrm{C}}\left(\mathrm{CDCl}_{3}\right) 167.1(\mathrm{C} 2), 141.5,137.3,136.0,135.1,128.5,128.5,128.0,127.4,126.9,126.5$, 125.2, $114.7\left(\mathrm{CH}=\mathrm{CH}_{2}\right)$, 59.8, 57.0, $52.6(\mathrm{NCH}), 22.4\left(\mathrm{CH}_{3}\right), 18.8\left(\mathrm{CH}_{3}\right) . \mathrm{IR}\left(\mathrm{CHCl}_{3}, \mathrm{~cm}^{-1}\right): v$ 1738 (NC=O). Anal. Calcd for $\mathrm{C}_{22} \mathrm{H}_{23} \mathrm{NO}$ : C, 83.24; H, 7.30; N, 4.41. Found: C, 83.46; H, 7.16; N, 4.55 .

Preparation of azocin-2-ones 5. A solution of the corresponding dialkenyl- $\beta$-lactam (1.0 mmol) in anhydrous toluene ( $5 \mathrm{~mL}$ ) was heated in a sealed tube at $120^{\circ} \mathrm{C}$ until complete disappearance of the starting material (TLC). The reaction was allowed to cool to room temperature, the solvent was removed under reduced pressure, and after purification by flash chromatography compounds 5 were obtained.

Azocin-2-one ( \pm -5a. From $70 \mathrm{mg}(0.24 \mathrm{mmol})$ of compound $( \pm)-2 \mathbf{a}, 60 \mathrm{mg}(86 \%)$ of compound ( \pm )-5a was obtained as a colourless oil after purification by flash chromatography (hexanes/ethyl acetate 5:1) NMR data: $\delta_{\mathrm{H}}\left(\mathrm{CDCl}_{3}\right) 2.34$ (bs, $\left.1 \mathrm{H}, J=14.0 \mathrm{~Hz}, \mathrm{H} 5\right), 2.75$ (bs, $1 \mathrm{H}$, $J=14.0 \mathrm{~Hz}, \mathrm{H} 5$ ), 3.69 (s, 3H, $\mathrm{OCH}_{3}$ ), 3.73 (s, 3H, $\mathrm{OCH}_{3}$ ), 3.75 (m, 1H, H6), 5.71 (dd, $1 \mathrm{H}, J=$ 7.6, $9.3 \mathrm{~Hz}, \mathrm{H7}$ ), 5.85 (ddd, 1H, $J=2.7,5.4,13.2 \mathrm{~Hz}, \mathrm{H} 4$ ), 6.09 (dt, $1 \mathrm{H}, J=2.2,13.2 \mathrm{~Hz}, \mathrm{H} 3$ ), 6.19 ( d, $1 \mathrm{H}, J=7.6 \mathrm{~Hz}, \mathrm{H} 8), 6.84(\mathrm{~m}, 2 \mathrm{H}, \mathrm{Ar}), 7.14(\mathrm{~m}, 2 \mathrm{H}, \mathrm{Ar}) . \delta_{\mathrm{C}}\left(\mathrm{CDCl}_{3}\right) 173.2(\mathrm{C}=\mathrm{O})$, 167.1 (C2), 158.3, 132.0, 131.0, 130.2, 127.2, 124.1, 123.4, 114.2, 55.4, 52.4, 39.4, 32.5. IR $\left(\mathrm{CHCl}_{3}, \mathrm{~cm}^{-1}\right): v 1735(\mathrm{NC}=\mathrm{O}), 1664(\mathrm{C}=\mathrm{O}), 1624,1607,1510,1248 . \mathrm{MS}(\mathrm{EI}), \mathrm{m} / z: 288\left(\mathrm{M}^{+}\right.$ 
+1, 24), $287\left(\mathrm{M}^{+}, 82\right), 228\left(\mathrm{M}^{+}\right.$-59, 97), 134 (100). Anal. Calcd for $\mathrm{C}_{16} \mathrm{H}_{17} \mathrm{NO}_{4}$ : C, 66.89; $\mathrm{H}$, 5.96; N, 4.88. Found: C, 66.96; H, 5.84; N, 4.99.

Azocin-2-one $( \pm)-5 \mathbf{b}$. From $33 \mathrm{mg}(0.11 \mathrm{mmol})$ of compound $( \pm)-2 \mathbf{b}, 30 \mathrm{mg}(89 \%)$ of compound $( \pm)$-5b was obtained as a colourless oil after purification by flash chromatography (hexanes/ethyl acetate 3:1). NMR data: $\delta_{\mathrm{H}}\left(\mathrm{DMSO}, 70{ }^{\circ} \mathrm{C}\right) 1.81\left(\mathrm{~s}, 3 \mathrm{H}, \mathrm{CH}_{3}\right), 2.41$ (bs, 1H, H5), 2.67 (bs, 1H, $J=15.6$ Hz, H5'), 3.63 (m, 1H, H6), 3.69 (s, 3H, $\mathrm{OCH}_{3}$ ), 3.76 (s, 3H, OCH $), 5.60$ (t, 1H, $J=8.1 \mathrm{~Hz}, \mathrm{H} 7), 5.84$ (bs, 1H, H3), 6.19 (d, 1H, $J=6.8 \mathrm{~Hz}, \mathrm{H8}$ ), 6.95 (m, 2H, Ar), 7.16 $(\mathrm{m}, 2 \mathrm{H}, \mathrm{Ar}) . \delta_{\mathrm{C}}\left(\mathrm{CDCl}_{3}\right) 176.1(\mathrm{C}=\mathrm{O}), 167.4(\mathrm{C} 2), 158.2,138.9,132.1,130.6,127.2,123.3$, 120.2, 114.1, 55.3, 52.2, 39.5, 37.2, $25.7\left(\mathrm{CH}_{3}\right)$. IR $\left(\mathrm{CHCl}_{3}, \mathrm{~cm}^{-1}\right): v 1736(\mathrm{NC}=\mathrm{O}), 1664$ (C=O), 1624, 1508, 1248. MS (EI), m/z: 301 ( $\left.\mathrm{M}^{+}, 40\right), 286$ (2), 242 (65), 134 (100). Anal. Calcd for $\mathrm{C}_{17} \mathrm{H}_{19} \mathrm{NO}_{4}$ : C, 67.76; H, 6.36; N, 4.65. Found: C, 67.57; H, 6.44; N, 4.49.

Azocin-2-one $( \pm)-5 c$. From $100 \mathrm{mg}(0.37 \mathrm{mmol})$ of compound $( \pm)-2 c, 85 \mathrm{mg}(85 \%)$ of compound $( \pm)-5 c$ was obtained as a white solid after purification by flash chromatography (hexanes/ethyl acetate 4:1). Mp $115-117{ }^{\circ} \mathrm{C}$ (hexanes/ethyl acetate). NMR data: $\delta_{\mathrm{H}}\left(\mathrm{CDCl}_{3}\right)$ 2.20 (m, 1H, H5), 2.21 (s, 3H, CH 3 ), 2.65 (bd, 1H, J=15.7 Hz, H5'), 3.75 (s, 3H, OCH ), 3.79 (m, 1H, H6), 5.55 (dd, 1H, $J=7.6,9.5$ Hz, H7), 5.81 (ddd, 1H, $J=2.9$, 5.4, $13.0 \mathrm{~Hz}, \mathrm{H} 4$ ), 6.02 (dt, 1H, $J=2.1,13.2 \mathrm{~Hz}, \mathrm{H} 3$ ), 6.16 (d, 1H, $J=7.6 \mathrm{~Hz}, \mathrm{H} 8$ ), 6.86 (m, 2H, Ar), 7.15 (m, 2H, Ar). $\delta_{\mathrm{C}}\left(\mathrm{CDCl}_{3}\right) 206.9(\mathrm{C}=\mathrm{O}), 167.4(\mathrm{C} 2), 158.4,132.1,131.6,130.6,127.1,124.3,123.3,114.4$, 55.5, 46.7, 31.4 (C5), $29.3\left(\mathrm{CH}_{3}\right)$. IR $\left(\mathrm{CHCl}_{3}, \mathrm{~cm}^{-1}\right): v 1707(\mathrm{NC}=\mathrm{O}), 1662(\mathrm{C}=\mathrm{O}), 1630,1512$, 1244. Anal. Calcd for $\mathrm{C}_{16} \mathrm{H}_{17} \mathrm{NO}_{3}$ : C, 70.83; H, 6.32; N, 5.16. Found: C, 70.67; H, 6.45; N, 5.29. Azocin-2-one $( \pm)-5 d$. From $60 \mathrm{mg}(0.21 \mathrm{mmol})$ of compound $( \pm)-2 d, 52 \mathrm{mg}(87 \%)$ of compound ( \pm )-5d was obtained as a colourless oil after purification by flash chromatography (hexanes/ethyl acetate 4:1). NMR data: $\delta_{\mathrm{H}}\left(\mathrm{CDCl}_{3}\right) 1.78$ (s, 3H, $\left.\mathrm{CH}_{3}\right), 2.10$ (bs, 1H, H5), 2.20 (s, $3 \mathrm{H}, \mathrm{COCH}_{3}$ ), 2.59 (bd, 1H, $J=16.4 \mathrm{~Hz}, \mathrm{H} 5$ ), 3.74 (m, 1H, H6), 3.74 (s, 3H, $\mathrm{OCH}_{3}$ ), 5.54 (t, $1 \mathrm{H}, J=8.8 \mathrm{~Hz}, \mathrm{H} 7), 5.87$ (s, 1H, H3), 6.12 (d, 1H, $J=7.6 \mathrm{~Hz}, \mathrm{H} 8), 6.85$ (m, 2H, Ar), 7.13 (m, $2 \mathrm{H}, \mathrm{Ar}) . \delta_{\mathrm{C}}\left(\mathrm{CDCl}_{3}\right) 207.1(\mathrm{C}=\mathrm{O}), 167.8(\mathrm{C} 2), 158.3,139.5,132.2,131.3,127.1,123.4,120.3$, 114.3, $55.4\left(\mathrm{OCH}_{3}\right)$, 50.0, 35.9, $29.6\left(\mathrm{CH}_{3}\right), 29.2\left(\mathrm{CH}_{3}\right)$. IR $\left(\mathrm{CHCl}_{3}, \mathrm{~cm}^{-1}\right): v 1714(\mathrm{NC}=\mathrm{O})$, $1668(\mathrm{C}=\mathrm{O}), 1625,1604,1508,1245$. Anal. Calcd for $\mathrm{C}_{17} \mathrm{H}_{19} \mathrm{NO}_{3}$ : C, 78.97; H, 6.63; N, 4.39. Found: C, 78.76; H, 6.74; N, 4.41.

Azocin-2-one $( \pm)-5$ e. From $50 \mathrm{mg}(0.16 \mathrm{mmol})$ of compound cis- $( \pm)-2 \mathbf{e}, 35 \mathrm{mg}(70 \%)$ of compound $( \pm)$-5e was obtained as a colourless oil after purification by flash chromatography (hexanes/ethyl acetate 4:1). From compound trans-( \pm )-2e, $19 \mathrm{mg}$ (37\%) of compound ( \pm )-5e was obtained. NMR data: $\delta_{\mathrm{H}}\left(\mathrm{CDCl}_{3}\right) 2.50$ (bs, 1H, H5), 2.77 (bd, $1 \mathrm{H}, J=15.1 \mathrm{~Hz}, \mathrm{H} 5$ ), 3.81 (s, 3H, $\mathrm{OCH}_{3}$ ), 4.13 (bs, 1H, H6), 5.68 (t, 1H, $J=9.3 \mathrm{~Hz}, \mathrm{H} 7$ ), 5.94 (m, 1H, H4), 6.16 (m, 2H, H3, H8), 6.92 (m, 2H, Ar), 7.25 (m, 7H, Ar). $\delta_{\mathrm{C}}\left(\mathrm{CDCl}_{3}\right) 167.8$ (C2), 158.3, 142.7, 129.0, 129.0, 128.9, 127.3, 127.0, 127.0, 126.9, 126.8, 124.3, 114.3, $55.5\left(\mathrm{OCH}_{3}\right), 39.6,36.8(\mathrm{C} 5) . \mathrm{IR}\left(\mathrm{CHCl}_{3}, \mathrm{~cm}^{-1}\right)$ : v $1735(\mathrm{C}=\mathrm{O}), 1622(\mathrm{C}=\mathrm{O}), 1510$. MS (EI), m/z: $306\left(\mathrm{M}^{+}+1,23\right), 305\left(\mathrm{M}^{+}, 74\right), 236(100)$. Anal. Calcd for $\mathrm{C}_{20} \mathrm{H}_{19} \mathrm{NO}_{2}$ : C, 78.66; H, 6.27; N, 4.59. Found: C, 78.58; H, 6.14; N, 4.45.

Azocin-2-one ( \pm )-5f. From $100 \mathrm{mg}(0.31 \mathrm{mmol})$ of the mixture of isomers $( \pm)-2 \mathrm{f}, 60 \mathrm{mg}(60 \%)$ of compound $( \pm)$-5f was obtained as a colourless oil after purification by flash chromatography 
(hexanes/ethyl acetate 3:1). NMR data: $\delta_{\mathrm{H}}\left(\mathrm{CDCl}_{3}\right) 1.90$ (s, 3H, $\left.\mathrm{CH}_{3}\right), 2.35$ (bs, 1H, H5), 2.75 (bs, 1H, H5'), 3.81 (s, 3H, $\mathrm{OCH}_{3}$ ), 4.11 (bs, 1H, H6), 5.69 (bs, 1H, H7), 5.99 (s, 1H, H3), 6.10 (d, $1 \mathrm{H}, J=7.8 \mathrm{~Hz}, \mathrm{H} 8), 6.92(\mathrm{~m}, 2 \mathrm{H}, \mathrm{PMP}), 7.20-7.40$ (m, 7H, Ar). $\delta_{\mathrm{C}}\left(\mathrm{CDCl}_{3}\right) 168.2(\mathrm{C} 2)$, 158.3, 142.7, 140.5, 132.6, 129.5, 128.9, 128.9, 128.6, 127.2, 127.0, 120.3, 114.3, $55.5\left(\mathrm{OCH}_{3}\right)$, 41.9, 39.2, 26.2. IR $\left(\mathrm{CHCl}_{3}, \mathrm{~cm}^{-1}\right): v 1710(\mathrm{C}=\mathrm{O}), 1664,1622,1508,1246 . \mathrm{MS}(\mathrm{EI}), \mathrm{m} / z: 320$ $\left(\mathrm{M}^{+}+1,28\right), 319\left(\mathrm{M}^{+}, 82\right), 304\left(\mathrm{M}^{+}-15,24\right), 236(100)$. Anal. Calcd for $\mathrm{C}_{21} \mathrm{H}_{21} \mathrm{NO}_{2}$ : C, 78.97; H, 6.63; N, 4.39. Found: C, 79.09; H, 6.55; N, 4.52.

Azocin-2-one $( \pm)-5 g$. From $51 \mathrm{mg}(0.20 \mathrm{mmol})$ of compound $E-( \pm)-2 \mathrm{~g}, 51 \mathrm{mg}$ (quantitative yield) of compound ( \pm )-5g was obtained as a white solid after purification by flash chromatography (hexanes/ethyl acetate 3:1). From Z-( \pm )-2g, $51 \mathrm{mg}$ (quantitative yield) of compound ( \pm )-5g was obtained. Mp $135-137^{\circ} \mathrm{C}$ (hexanes/ethyl acetate). NMR data: $\delta_{\mathrm{H}}\left(\mathrm{CDCl}_{3}\right)$ 2.56 (dddd, $1 \mathrm{H}, J=1.5,5.4,12.7,18.3 \mathrm{~Hz}, \mathrm{H} 5$ ), 2.94 (ddt, 1H, $J=2.4,4.4,18.3 \mathrm{~Hz}, \mathrm{H} 5$ '), 3.82 (s, 3H, $\mathrm{OCH}_{3}$ ), 3.95 (ddd, $1 \mathrm{H}, J=4.1,9.3,12.7 \mathrm{~Hz}, \mathrm{H6}$ ), 5.59 (dd, 1H, $J=7.6,9.3 \mathrm{~Hz}, \mathrm{H} 7$ ), 5.81 (ddd, $1 \mathrm{H}, J=2.9,5.4,13.1 \mathrm{~Hz}, \mathrm{H} 4), 6.13$ (dt, 1H, $J=2.0,13.1 \mathrm{~Hz}, \mathrm{H} 3$ ), 6.28 (dd, $1 \mathrm{H}, J=1.0$, $7.6 \mathrm{~Hz}, \mathrm{H} 8), 6.94$ (m, 2H, Ar), 7.19 (m, 2H, Ar). $\delta_{\mathrm{C}}\left(\mathrm{CDCl}_{3}\right) 166.3$ (C2), 158.8, 132.8 (C4), 131.4, 127.9 (C8), 127.2, 124.9 (C3), 124.8 (CN), 119.5 (C7), 114.5, $55.5\left(\mathrm{OCH}_{3}\right), 33.3$ (C5), 25.7 (C6). MS (EI), m/z: $255\left(\mathrm{M}^{+}+1,17\right), 254\left(\mathrm{M}^{+}, 85\right), 134$ (100). IR $\left(\mathrm{KBr}, \mathrm{cm}^{-1}\right): v 1735$ $(\mathrm{C}=\mathrm{O})$. Anal. Calcd for $\mathrm{C}_{15} \mathrm{H}_{14} \mathrm{~N}_{2} \mathrm{O}_{2}$ : C, 70.85; H, 5.55; N, 11.02. Found: C, 70.90; H, 5.39; N, 10.98 .

Azocin-2-one $( \pm)-5 \mathbf{h}$. From $31 \mathrm{mg}(0.12 \mathrm{mmol})$ of compound $E-( \pm)-2 \mathbf{h}, 31 \mathrm{mg}$ (quantitative yield) of compound ( \pm )-5h was obtained as a white solid after flash chromatography (hexanes/ethyl acetate 3:1). From $Z-( \pm)-2 \mathbf{h}, 31 \mathrm{mg}$ of compound ( \pm )-5h (quantitative yield). Mp $162-164{ }^{\circ} \mathrm{C}$ (hexanes/ethyl acetate). NMR data: $\delta_{\mathrm{H}}\left(\mathrm{CDCl}_{3}\right) 1.86\left(\mathrm{~s}, 3 \mathrm{H}, \mathrm{CH}_{3}\right), 2.61$ (dd, $1 \mathrm{H}, J=$ 12.2, 16.6 Hz, H5), 2.85 (dd, 1H, $J=4.9,16.6 \mathrm{~Hz}, \mathrm{H} 5$ '), 3.79 (s, 3H, O CH 3 ), 4.06 (ddd, 1H, $J=$ 4.1, 8.3, $12.2 \mathrm{~Hz}, \mathrm{H} 6$ ), 5.58 (t, 1H, $J=8.1 \mathrm{~Hz}, \mathrm{H} 7$ ), 5.90 (bs, $1 \mathrm{H}, \mathrm{H} 3$ ), 6.30 (d, $1 \mathrm{H}, J=7.8 \mathrm{~Hz}$, $\mathrm{H} 8), 6.97(\mathrm{~m}, 2 \mathrm{H}, \mathrm{Ar}), 7.22(\mathrm{~m}, 2 \mathrm{H}, \mathrm{Ar}) . \delta_{\mathrm{C}}\left(\mathrm{CDCl}_{3}\right) 166.8$ (C2), 158.8, 137.2 (C4), 132.7 (C8), 131.6, 129.0 (C3), 127.3, 121.1 (C7), 119.5 (CN), 114.5, $55.5\left(\mathrm{O} \mathrm{CH}_{3}\right), 37.7$ (C5), $26.0\left(\mathrm{CH}_{3}\right)$, 25.3 (C6). IR $\left(\mathrm{CHCl}_{3}, \mathrm{~cm}^{-1}\right): v 1735(\mathrm{C}=\mathrm{O})$. MS (EI), m/z: $269\left(\mathrm{M}^{+}+1,17\right), 268\left(\mathrm{M}^{+}, 78\right), 253$ $\left(\mathrm{M}^{+}-15,10\right), 134$ (100). Anal. Calcd for $\mathrm{C}_{16} \mathrm{H}_{16} \mathrm{~N}_{2} \mathrm{O}_{2}$ : C, 71.62; H, 6.01; N, 10.44. Found: C, 71.55; H, 6.11; N, 10.39 .

Azocin-2-one ( \pm )-5i. From $210 \mathrm{mg}(0.73 \mathrm{mmol})$ of compound $( \pm)-3 a, 165 \mathrm{mg}(79 \%)$ of compound $( \pm$ )-5i was obtained as a white solid after flash chromatography (hexanes/ethyl acetate 5:1). Mp 73-75 ${ }^{\circ} \mathrm{C}$ (hexanes/ethyl acetate). NMR data: $\delta_{\mathrm{H}}\left(\mathrm{CDCl}_{3}\right) 2.44$ (bs, $\left.1 \mathrm{H}, \mathrm{H} 5\right), 2.60$ (bs, 1H, H5'), 3.56 (bs, 1H, H6), 4.09 (d, 1H, $J=11.5 \mathrm{~Hz}, \mathrm{NCHH}), 5.32$ (m, 1H, NCHH) , 5.44 (t, $1 \mathrm{H}, J=7.8 \mathrm{~Hz}, \mathrm{H} 7$ ), 5.80-6.10 (m, 3H, H3, H4, H8), 6.69 (bs, 2H, Ar), 7.10-7.50 (m, 8H, Ar). $\delta_{\mathrm{C}}\left(\mathrm{CDCl}_{3}\right) 167.4(\mathrm{C} 2), 142.3,136.5,132.0,130.8,129.1,128.6,128.4,127.6,126.8,126.6$, 125.2, 123.7, 50.3, 39.1, 35.9. IR (KBr, $\left.\mathrm{cm}^{-1}\right): v 1656(\mathrm{C}=\mathrm{O}), 1612,1598,1492,1423,1259$. Anal. Calcd for $\mathrm{C}_{20} \mathrm{H}_{19} \mathrm{NO}$ : C, 83.01; H, 6.62; N, 4.84. Found: C, 82.92; H, 6.70; N, 4.63.

Azocin-2-one ( \pm -5j. From $194 \mathrm{mg}(0.63 \mathrm{mmol})$ of compound $( \pm)-3 \mathbf{b}, 155 \mathrm{mg}(80 \%)$ of compound $( \pm)-5 \mathbf{j}$ was obtained as a white solid after flash chromatography (hexanes/ethyl acetate 
5:1). Mp 109-111 ${ }^{\circ} \mathrm{C}$ (hexanes/ethyl acetate). NMR data: $\delta_{\mathrm{H}}\left(\mathrm{CDCl}_{3}\right) 1.80\left(\mathrm{~s}, 3 \mathrm{H}, \mathrm{CH}_{3}\right), 2.33$ (d, $1 \mathrm{H}, J=13.9 \mathrm{~Hz}, \mathrm{H} 5), 2.51$ (m, 1H, H5'), 3.52 (m, 1H, H6), 4.67 (m, 2H, NCH${ }_{2} \mathrm{Ph}$ ), 5.38 (t, 1H, $J$ = $8.1 \mathrm{~Hz}, \mathrm{H} 7), 5.80$ (s, 1H, H3), 6.05 (d, 1H, $J=8.1 \mathrm{~Hz}, \mathrm{H} 8), 6.85$ (m, 2H, Ar), 7.15 (m, 3H, $\mathrm{Ar}), 7.30(\mathrm{~m}, 5 \mathrm{H}, \mathrm{Ar}) . \delta_{\mathrm{C}}\left(\mathrm{CDCl}_{3}\right) 167.5(\mathrm{C} 2), 142.4,140.5,136.6,130.6,129.1,128.6,128.4$, 128.4, 127.5, 126.8, 126.5, 119.9, $50.2\left(\mathrm{NCH}_{2}\right)$, 41.0, 39.2, 26.3. IR $\left(\mathrm{CHCl}_{3}, \mathrm{~cm}^{-1}\right): v 1728$ $(\mathrm{NC}=\mathrm{O}), 1662(\mathrm{C}=\mathrm{O}), 1610,1255$. Anal. Calcd for $\mathrm{C}_{21} \mathrm{H}_{21} \mathrm{NO}$ : C, 83.13; H, 6.98; N, 4.62. Found: C, 83.02; H, 7.10; N, 4.59.

Azocin-2-one (-)-5k- $\alpha$. From $110 \mathrm{mg}(0.36 \mathrm{mmol})$ of compound (+)-4a- $\alpha, 90 \mathrm{mg}(82 \%)$ of compound (-)-5k- $\alpha$ was obtained as a colourless oil after flash chromatography (hexanes/ethyl acetate 5:1). $[\alpha]_{\mathrm{D}}=-188\left(c 1.0, \mathrm{CHCl}_{3}\right)$. NMR data: $\delta_{\mathrm{H}}\left(\mathrm{CDCl}_{3}\right) 1.58\left(\mathrm{~d}, 3 \mathrm{H}, J=7.6 \mathrm{~Hz}, \mathrm{CH}_{3}\right)$, 2.42 (m, 1H, H5), 2.74 (bd, 1H, $J=18.6 \mathrm{~Hz}, \mathrm{H} 5$ ), 3.97 (m, 1H, H6), 5.61 (m, 2H, $\mathrm{CH}_{3} \mathrm{CHPh}$, H7), 5.84 (m, 1H, H4), 5.99-6.07 (m, 1H, H3), 6.12 (q, 1H, J = 7.6 Hz, H8), 7.32 (m, 10H, Ar). $\delta_{\mathrm{C}}\left(\mathrm{CDCl}_{3}\right) 167.2(\mathrm{C} 2), 142.6,139.0,131.5,131.2,128.7,128.3,127.6,127.5,127.1,126.8$, 124.5, 124.0, 51.1, 39.2, 36.5, 16.0. IR $\left(\mathrm{CHCl}_{3}, \mathrm{~cm}^{-1}\right): v 1743(\mathrm{C}=\mathrm{O}), 1712,1658,1601 . \mathrm{MS}$ (EI), $m / z$ : $304\left(\mathrm{M}^{+}+1,3\right), 303\left(\mathrm{M}^{+}, 1\right), 288$ (1), 105 (100). Anal. Calcd for $\mathrm{C}_{21} \mathrm{H}_{21} \mathrm{NO}$ : C, 83.13; H, 6.98; N, 4.62. Found: C, 83.22; H, 6.70; N, 4.59.

Azocin-2-one (+)-5k- $\beta$. From $50 \mathrm{mg}(0.16 \mathrm{mmol})$ of compound (+)-4a- $\beta, 40 \mathrm{mg}(75 \%)$ of compound (+)-5k- $\boldsymbol{\beta}$ was obtained as a colourless oil after flash chromatography (hexanes/ethyl acetate 5:1). $[\alpha]_{\mathrm{D}}=+155\left(c 1.0, \mathrm{CHCl}_{3}\right)$. NMR data: $\delta_{\mathrm{H}}\left(\mathrm{CDCl}_{3}\right) 1.59$ (d, 3H, $\left.J=7.8 \mathrm{~Hz}, \mathrm{CH}_{3}\right)$, 2.33 (m, 1H, H5), 2.58 (bd, 1H, $J=17.8 \mathrm{~Hz}, \mathrm{H} 5$ ) , 3.38 (m, 1H, H6), 5.46 (t, 1H, $J=9.5 \mathrm{~Hz}$, H7), 5.80 (m, 1H, H4), 5.95-6.13 (m, 3H, H3, H8, CH $\mathrm{CHPh}_{3}, 6.60$ (m, 2H, Ar), 7.15-7.45 (m, $8 \mathrm{H}, \mathrm{Ar}) . \delta_{\mathrm{C}}\left(\mathrm{CDCl}_{3}\right)$ 167.2 (C2), 142.3, 132.9, 131.3, 128.4, 128.3, 127.7, 127.5, 127.1, 127.0, 126.6, 124.2, 123.8, 51.7, 38.8, 35.8, 15.9. IR $\left(\mathrm{CHCl}_{3}, \mathrm{~cm}^{-1}\right): v 1735(\mathrm{C}=\mathrm{O}), 1718,1659,1601$. Anal. Calcd for $\mathrm{C}_{21} \mathrm{H}_{21} \mathrm{NO}$ : C, 83.13; H, 6.98; N, 4.62. Found: C, 83.25; H, 6.81; N, 4.53.

Azocin-2-one (-)-5l- $\alpha$. From $80 \mathrm{mg}(0.25 \mathrm{mmol})$ of compound (-)-4b- $\alpha, 60 \mathrm{mg}$ (75\%) of compound (-)-5l- $\alpha$ was obtained as a colourless oil after flash chromatography (hexanes/ethyl acetate 5:1). $[\alpha]_{\mathrm{D}}=-147\left(c 1.1, \mathrm{CHCl}_{3}\right)$. NMR data: $\delta_{\mathrm{H}}\left(\mathrm{CDCl}_{3}\right) 1.57(\mathrm{~d}, 3 \mathrm{H}, J=6.8 \mathrm{~Hz}$, $\mathrm{PhCHCH}_{3}$ ), 1.83 (s, 3H, CH 3 ), 2.26 (dd, $1 \mathrm{H}, J=13.1,17.5 \mathrm{~Hz}, \mathrm{H}$ ), 2.71 (bd, $1 \mathrm{H}, J=17.5 \mathrm{~Hz}$, H5'), 3.95 (m, 1H, H6), 5.60 (m, 2H, NCHPh, H7), 5.88 (d, 1H, $J=1.2 \mathrm{~Hz}, \mathrm{H} 3$ ), 6.11 (q, 1H, $J=$ $6.8 \mathrm{~Hz}, \mathrm{H} 8), 7.26$ (m, 10H, Ar). $\delta_{\mathrm{C}}\left(\mathrm{CDCl}_{3}\right) 167.5$ (C2), 142.8, 140.0, 139.3, 132.2, 131.5, 128.8, 128.4, 127.6, 127.6, 126.9, 124.4, 120.5, 51.2, 41.7, 39.5, 26.1, 16.1. . IR $\left(\mathrm{CHCl}_{3}, \mathrm{~cm}^{-1}\right): v 1739$ $(\mathrm{C}=\mathrm{O}), 1650$, 1600. Anal. Calcd for $\mathrm{C}_{22} \mathrm{H}_{23} \mathrm{NO}$ : C, 83.24; $\mathrm{H}, 7.30 ; \mathrm{N}, 4.41$. Found: C, 83.17; H, 7.40; N, 4.55.

Azocin-2-one (+)-5l- $\beta$. From $30 \mathrm{mg}(0.09 \mathrm{mmol})$ of compound (+)-4b- $\beta, 25 \mathrm{mg}(83 \%)$ of compound (+)-5l- $\beta$ was obtained as a colourless oil after flash chromatography (hexanes/ethyl acetate 5:1). $[\alpha]_{\mathrm{D}}=+166\left(c\right.$ 1.5, $\left.\mathrm{CHCl}_{3}\right)$. NMR data: $\delta_{\mathrm{H}}\left(\mathrm{CDCl}_{3}\right) 1.62(\mathrm{~d}, 3 \mathrm{H}, J=6.8 \mathrm{~Hz}$, $\mathrm{PhCHCH}_{3}$ ), 1.82 (s, 3H, $\mathrm{CH}_{3}$ ), 2.18 (dd, 1H, $J=13.2,16.9 \mathrm{~Hz}, \mathrm{CHH}$ ), 2.57 (bd., $1 \mathrm{H}, J=16.9$ $\mathrm{Hz}, \mathrm{CH} H$ ), 3.41 (m, 1H, CH-Ph), 5.48 (t, 1H, $J=9.1 \mathrm{~Hz}, \mathrm{CH}=\mathrm{CH}-\mathrm{N}), 5.83$ (s, 1H, NCHPh), 6.09-6.19 (m, 2H, $\left.\mathrm{CH}_{3} \mathrm{C}=\mathrm{CH}, \mathrm{CH}=\mathrm{CH}-\mathrm{N}\right), 6.62(\mathrm{~m}, 2 \mathrm{H}, \mathrm{Ar}), 7.16-7.44(\mathrm{~m}, 8 \mathrm{H}, \mathrm{Ar}) . \delta_{\mathrm{C}}$ $\left(\mathrm{CDCl}_{3}\right) 167.5(\mathrm{C}=\mathrm{O}), 142.5,132.2,128.4,127.7,127.4,126.9,126.5,126.2,123.6,122.2$, 
121.0, 120.4, 51.7, 41.0, 39.0, 26.3, 16.0. IR $\left(\mathrm{CHCl}_{3}, \mathrm{~cm}^{-1}\right): v 1740(\mathrm{C}=\mathrm{O}), 1650$, 1605. Anal. Calcd for $\mathrm{C}_{22} \mathrm{H}_{23} \mathrm{NO}$ : C, 83.24; H, 7.30; N, 4.41. Found: C, 83.19; H, 7.23; N, 4.22.

\section{Acknowledgements}

Support for this work by the DGI-MEC (CTQ2006-10292), Comunidad Autónoma de Madrid (CCG-07-UCM/PPQ-2308), and Universidad Complutense de Madrid (Grant GR74/07) are gratefully acknowledged.

\section{References}

1. For selected examples, see: Basil, B.; Coffee, E. C. J.; Gell, D. L.; Maxwell, D. R.; Sheffield, D. J.; Wooldridge, K. R. H. J. Med. Chem. 1970, 13, 403. Klayman, D. L.; Scovill, J. P.; Bartosevich, J. F.; Mason, C. J. J. Med. Chem. 1979, 22, 1367. Vedejs, E.; Galante, R. J.; Goekjian, P. G. J. Am. Chem. Soc. 1998, 120, 3613.

2. Pflantz, R.; Tielmann, P.; Rössle, M.; Hoenke, C.; Christoffers, J. Eur. J. Org. Chem. 2007, 3227. Pearson, W. H.; Lee , I. Y.; Mi , Y.; Stoy, P. J. Org. Chem. 2004 , 69, 9109. Arya, P.; Couve-Bonnaire, S.; Durieux, P.; Laforce, D.; Kumar, R.; Leek, D. M. J. Comb. Chem. 2004, 6, 735. Hansen, L. K.; Størmer, F. C.; Petersen, D.; Aasen, A. J. Acta Crystallogr. Sec. E 2001, 57, 909.

3. Bergemann, S.; Brecht, R.; Büttner, F.; Guenard, D.; Gust, R.; Seitz, G.; Stubbs, M. T.; Thoret, S. Bioorg. Med. Chem. 2003, 11, 1269. Baudoin, O.; Cesario, M.; Guenard, D.; Gueritte, F. J. Org. Chem. 2002, 67, 1199. Brecht, R.; Seitz, G.; Guenard, D.; Thoret, S. Bioorg. Med. Chem. 2000, 8, 557. Berg, U.; Bladh, H.; Svensson, C.; Wallin, M. Bioorg. Med. Chem. Lett. 1997, 7, 2771. Schoen, W. R.; Pisano, J. M.; Prandergast, K.; Wyvratt, M. J.; Fisher, M. H.; Cheng, K.; Chan, W. W.-S.; Butler, B.; Smith, R. G.; Ball, R. G. J. Med. Chem. 1994, 37, 897. Watthey, J. W. H.; Stanton, J. L.; Desai, M.; Babiarz, J. E.; Finn, B. M. J. Med. Chem. 1985, 28, 1511.

4. Kaul, R.; Surprenant, S.; Lubell, W. D. J. Org. Chem. 2005, 70, 4901. Creighton, C. J.; Leo, G. C.; Du, Y.; Reitz, A. B. Bioorg. Med. Chem. 2004, 12, 4375. Derrer, S.; Davies, J. E.; Holmes, A. B. J. Chem. Soc., Perkin Trans. 1 2000, 2943. Derrer, S.; Davies, J. E.; Holmes, A. B. J. Chem. Soc., Perkin Trans. 1 2000, 2957. Derrer, S.; Feeder, N.; Teat, S. J.; Davies, J. E.; Holmes, A. B. Tetrahedron Lett. 1998, 39, 9309.

5. For reviews, see: Galli, C.; Mandolini, L. Eur. J. Org. Chem. 2000, 3117. Illuminati, G.; Mandolini, L. Acc. Chem. Res. 1981, 14, 95.

6. For reviews, see: Nubbemeyer, U. Synlett 2003, 961. Allin, S. M.; Baird, R. D. Curr. Org. Chem. 2003, 5, 395. Hill, R. K. Comprehensive Organic Synthesis, Pergamon: Oxford, 1991, Vol.5, p 785. Bronson, J. J.; Danheiser, R. L. Comprehensive Organic Synthesis, Pergamon: Oxford, 1991, Vol. 5, p 999. 
7. See, for example: Setti, E. L.; Micetich, R. G. Curr. Med. Chem. 1998, 5, 101. The Organic Chemistry of $\beta$-Lactams; Georg, G. I., Ed.; VCH: New York, 1993. Neuhaus, F. C.; Georgeopapadakou, N. H. In Emerging Targets in Antibacterial and Antifungal Chemoterapy; Sutcliffe, J.; Georgeopapadakou, N. H., Eds.; Chapman and Hall: New York, 1992. The Chemistry of $\beta$-Lactams; Page, M. I., Ed.; Chapman and Hall: London, 1992.

8. Some of the more notable advances concern the development of mechanism-based serine protease inhibitors of elastase, cytomegalovirus protease, thrombin, prostate specific antigen, and cell metastasis and as inhibitors of acyl-CoA cholesterol acyl transferase. For reviews, see: Veinberg, G.; Vorona, M.; Shestakova, I.; Kanepe, I.; Lukevics, E. Curr. Med. Chem. 2003, 10, 1741. Clader, J. W. J. Med. Chem. 2004, 47, 1. For selected examples, see: Kvaerno, L.; Ritter, T.; Werder, M.; Hauser, H.; Carreira, E. M. Ang. Chem. Int. Ed. 2004, 43, 4653. Burnett, D. A. Curr. Med. Chem. 2004, 11, 1873. (e) Page, M. I.; Laws, A. P. Tetrahedron 2000, 56, 5631. Haley, T. M.; Angier, S. J.; Borthwick, A. D.; Singh, R.; Micetich, R. G. Drugs 2000, 3, 512.

9. Rothstein, J. D.; Patel, S.; Regan, M. R.; Haenggeli, C.; Huang, Y. H.; Bergles, D. E.; Jin, L.; Hoberg, M. D.; Vidensky, S.; Chung, D. S.; Toan, S. V.; Bruijn, L. I.; Su, Z.-Z.; Gupta, P.; Fisher, P. B. Nature 2005, 433, 73.

10. For reviews, see: Alcaide, B.; Almendros, P.; Aragoncillo, C. Chem. Rev. 2007, 107, 4437. Alcaide, B.; Almendros, P. Curr. Med. Chem. 2004, 11, 1921. Deshmukh, A. R. A. S.; Bhawal, B. M.; Krishnaswamy, D.; Govande, V. V.; Shinkre, B. A.; Jayanthi, A. Curr. Med. Chem. 2004, 11, 1889. Alcaide, B.; Almendros, P. Synlett 2002, 381. Palomo, C.; Aizpurua, J. M.; Ganboa, I.; Oiarbide, M. Synlett 2001, 1813. Alcaide, B.; Almendros, P. Org. Prep. Proced. Int. 2001, 33, 315. Palomo, C.; Aizpurua, J. M.; Ganboa, I.; Oiarbide, M. Aminoacids, 1999, 16, 321. Ojima, I.; Delaloge, F. Chem. Soc. Rev. 1997, 26, 377. Ojima, I. Adv. Asym. Synth. 1995, 1, 95. Manhas, M. S.; Wagle, D. R.; Chiang, J.; Bose, A. K. Heterocycles 1988, 27, 1755.

11. For a thermal cis/trans isomerization in 2-azetidinones, see: Alcaide, B.; Almendros, P.; Salgado, N. R.; Rodríguez-Vicente, A. J. Org. Chem., 2000, 65, 4453. For thermal fragmentations of $\beta$-lactams, see: Paquette, L. A.; Wyvratt, M. J.; Allen Jr, G. R. J. Am. Chem. Soc. 1970, 92, 1763. Kappe, C. O.; Kollenz, G.; Netsch, K.-P.; Leung-Toung, R.; Wentrup, C. Chem. Commun. 1992, 488.

12. See, for example: Alcaide, B.; Almendros, P.; Martínez del Campo, T. Chem. Eur. J. 2008, 14, 7756. Alcaide, B.; Almendros, P.; Carrascosa, R.; Redondo, M. C. Chem. Eur. J. 2008, 14, 637. Alcaide, B.; Almendros, P.; Martínez del Campo, T. Angew. Chem. Int. Ed. 2007, 46, 6684. Alcaide, B.; Almendros, P.; Cabrero, G.; Ruiz, M. P. Chem. Commun. 2007, 4788. Alcaide, B.; Almendros, P.; Aragoncillo, C., Redondo, M. C. J. Org. Chem. 2007, 72, 1604. Alcaide, B.; Almendros, P.; Martínez del Campo, T. Angew. Chem. Int. Ed. 2006, 45, 4501. Alcaide, B.; Almendros, P.; Alonso, J. M. Chem. Eur. J. 2006, 12, 2874. Alcaide, B.; Almendros, P.; Aragoncillo, C.; Redondo, M. C.; Torres, M. R. Chem. Eur. J. 2006, 12, 1539. 
13. For a preliminary communication of a part of this work, see: Alcaide, B.; Rodríguez-Ranera, C.; Rodríguez-Vicente, A. Tetrahedron Lett. 2001, 42, 3081.

14. Alcaide, B.; Almendros, P. Chem. Soc. Rev. 2001, 30, 226.

15. Manhas, M. S.; Ghosh, M.; Bose, A. K. J. Org. Chem., 1990, 55, 575, and references cited therein.

16. Palomo, C.; Cossio, F. P.; Arrieta, A.; Odriozola, J. M.; Oiarbide, M.; Ontoria, J. M. J. Org. Chem. 1989, 54, 5736 and references cited therein.

17. Berson, J. A.; Dervan, P. B.; Malherbe, R.; Jenkins, J. A. J. Am. Chem. Soc. 1976, 98, 5937 and references cited therein. 